\title{
On the Separation of Enantiomers by Drift Tube Ion Mobility Spectrometry
}

\author{
Roberto Fernández-Maestre,,$^{1,2, \& *}$ and Markus Doerr ${ }^{3}$ \\ ${ }^{1}$ Departamento de Quimica, Campus de San Pablo, Universidad de Cartagena, Cartagena, \\ Colombia. \\ ${ }^{2}$ National Research Nuclear University MEPhI (Moscow Engineering Physics Institute), \\ Kashirskoe sh. 31, Moscow, 115409, Russia.*rfernandezm@unicartagena.edu.co \\ 3 Universidad Industrial de Santander, Cra 27 Calle 9, Bucaramanga, Colombia. \\ mhodoerr@uis.edu.co
}

\begin{abstract}
Racemic mixtures of twelve common $\alpha$-amino acids and three chiral drugs were tested for the separation of their enantiomers by ion mobility spectrometry (IMS)-quadrupole mass spectrometry (MS). Separations were tested by introducing chiral selectors in the mobility spectrometer buffer gas. (R)- $\alpha$-(trifluoromethyl) benzyl alcohol, (R)-tetrahydrofuran-2carbonitrile, (L)-ethyl lactate, methyl (S)-2-chloropropionate, and the R and S enantiomers of 2-butanol and 1-phenyl ethanol were evaluated as chiral selectors. Experimental conditions were varied during the tests including buffer gas temperature, concentration, and type of chiral selectors, analyte concentration, electrospray voltage, electrospray (ESI) solvent $\mathrm{pH}$, and buffer gas flow. The individual enantiomers yielded different drift times for periods of up to 8 hours in a few experiments; such drift times were sufficiently different ( $0.3 \mathrm{~ms}$ ) to partially resolve the enantiomers in racemic mixtures, but these mixtures always yielded a single mobility peak at the experimental conditions tested with a drift time similar to that of one of the enantiomers. Energy calculations of the chiral selector -ion interactions showed that these separations are unlikely using 2-butanol as chiral selector but they might be feasible depending on the nature of chiral selectors and the type of enantiomers.
\end{abstract}

Keywords: enantiomer separation, 2-butanol, theoretical calculations, racemic mixtures, ion mobility spectrometry

\& Department of Chemistry Fulmer Hall 630. Washington State University Pullman, WA 99164-4630 
Enantiomers are molecules that are non-superimposable mirror images of one another. Chiral compounds (enantiomers) are of significance because of their environmental impact and their economic, clinical, industrial, and biochemical importance. An indication of this importance is the homochirality requirement in all biological polymers of eukaryotes to meet their biological functions. ${ }^{1}$ Also, stereoselectivity is important in molecular recognition processes to explain the mechanisms behind the specific and different reaction rates of enantiomers.

The most widely accepted model for stereoselectivity is the three-point interactions model which calls for three-point interactions between receptor and substrate, the Pirkle Rule. ${ }^{2,3}$ In this model, stereochemical differences in biological activities are due to the differential binding of enantiomers to a common site on a receptor surface. To distinguish between enantiomers, the receptor must have three non-equivalent binding sites. Chiral discrimination occurs when one isomer can simultaneously interact with all sites whereas the other one interacts only with two sites. Steric factors and other non-binding or even repulsive interactions contribute to chiral discrimination. The three-point interaction model has been extensively applied and debated. ${ }^{4,5}$

Because enantiomers are so similar in structure, they are difficult to analyze, yet their analysis and purification are important in the pharmaceutical industry for the production of drugs which require a specific enantiomer. This is important because, very often, one enantiomer of the chiral drugs, the eutomer, is more active than the other, the distomer. The distomer may be inert or even toxic; ${ }^{6}$ in the thalidomide disaster in the 50's and 60's, the distomer of this racemic drug induced teratogenesis in fetuses when administered to pregnant women. ${ }^{7}$

The most common analytical method for the analysis and purification of enantiomers is chromatography. Enantiomers can be separated using a stereoselective stationary phase if one of the enantiomers is more attracted to the phase than the other. Examples of recent enantioseparations using chromatography are the separations of dansyl-DL-amino acids ${ }^{8}$ and enantiomers of baclofen and phenylalanine. ${ }^{9}$

Electrophoresis is another type of analytical method that has been used to separate enantiomers. ${ }^{10}$ For example, a chiral open tubular capillary column was made with sulfobutylether- $\beta$-cyclodextrin and glycidyl methacrylate for enantiomer separation in capillary electrochromatography. In these experiments, the separation of amlodipine besilate, 2,3-diphenylpropionic acid, tropic acid, and pantoprazole enantiomers was reported. ${ }^{11}$ Also, six mono- and di-tetraalkylammonium 1-tartrate ionic liquids with alkanes of different chain lengths were studied as chiral selectors in capillary electrophoresis. These chiral selectors showed an improved enantioseparation for ten commercial chiral drugs. ${ }^{12}$

The development of rapid methods for chiral compounds has been investigated because traditional methods for chiral separation, such as high-performance liquid chromatography and gas chromatography, are limited by long sample preparation and analysis times ${ }^{13}$ and the lack of enough resolution and sensitivity ${ }^{14}$. Mass spectrometry (MS) is the most widespread analytical method used for the rapid determination of the purity of chiral compounds. MS is not used as a separation technique, but as an indirect probe for chirality. With MS methods, enantiomers are not physically separated, but rather the difference in fragmentation patterns 
when the enantiomers are subjected to collisions with stereoselective agents is used to distinguish the chiral and even quantify components of a mixture. For example, diastereomeric adducts of chiral compounds with chiral host molecules, such as cyclodextrins (CDs) and crown ethers were investigated in single-stage MS experiments. ${ }^{15}$ It is clear from this and other examples that mass spectrometry is not an enantiomeric separation technique, but rather diastereomeric. ${ }^{16} \mathrm{MS}$ methods for chiral recognition are reviewed by Tao and Cooks, ${ }^{16}$ and $\mathrm{Wu}$ et al. ${ }^{17} \mathrm{MS}$ methods have a number of limitations as an indirect probe for chirality. These inherent limitations include the requirement of enantiopure reference compounds, cumbersome calibration procedures, non-standard instrument modifications, theoretical modeling of the ion fragmentation patterns, and compound-dependent enantiomeric selectivity. ${ }^{18}$

Another rapid analytical method that has been used for chiral separations is ion mobility spectrometry (IMS). IMS chiral separations can be fast, sensitive, and cheap because IMS spectra can be obtained in less than one minute, concentrations down to nmoles per liter can be detected and the instrumentation does not require high-cost vacuum pumps given that IMS is an atmospheric pressure technique. IMS separates gas-phase ions on the basis of their size and shape and thus has been used for the separation and analysis of stereoisomers in both stand-alone instruments or coupled with chromatography and mass spectrometry. Rister and Dodds reviewed the current literature on IMS analysis of steroids and steroid stereoisomers by stand-alone IMS, chromatography-IMS, and IMS-MS. ${ }^{19}$

The separation and analysis of enantiomers have been questionable by IMS. IMS was first applied to the separation of enantiomers by Dwivedi et al. in 2006. They reported the separation of racemic mixtures of amino acids by introducing (S) and (R)-2-butanol chiral selectors in the buffer gas and suggested that the enantiomer with the weaker interaction energy with the chiral selector would drift faster than the other one, leading to chiral separation. ${ }^{20}$ Karas patented this idea and claimed the separation of fluoxetine enantiomers using chiral 2-butanol. ${ }^{21}$ These enantiomer separations have not been reproduced at lower pressures, most common in commercial IMS instruments due to their greater sensitivity, maybe because of differences in clustering and related interactions. ${ }^{14}$ The same can be said for experiments at atmospheric pressure. Herbert Hill, research director of the IMS group in Washigton State University (USA), in a personal communication (2021) to FernandezMaestre, wrote that they "tried a number of times and were never able to reproduce" the chiral separations of the 2006 experiments. ${ }^{20}$ This comprised the studies of several graduate students, including Roscioli (2012). In Roscioli's study, methionine and methyl $\alpha$ glucopyranoside enantiomers were assayed for separation with the introduction of $(\mathrm{S})$ and (R)-2-butanol into the buffer gas of an ESI-IMS-tof-MS, reagents used by Dwivedi in 2006. They concluded that the separation of enantiomers with chiral modifiers was not possible using Dwivedi's experimental protocol. ${ }^{22}$

Additional reported IMS chiral separations have only been applied to the resolution of diastereomers, common in the literature because they have different physical properties in contrast to enantiomers and, therefore, are easier to separate. ${ }^{23}$ For example, the separation of $(\mathrm{R}, \mathrm{S})$ and $(\mathrm{S}, \mathrm{R})$-ephedrine and $(\mathrm{S}, \mathrm{S})$ and $(\mathrm{R}, \mathrm{R})$-pseudoephedrine diastereomers was achieved using IMS. Straight-chain achiral alcohols from methanol to n-octanol were used as drift gas chiral selectors. Clustering of alcohols to analytes induced conformational 
differences for $(\mathrm{R}, \mathrm{S})$-ephedrine and $(\mathrm{S}, \mathrm{S})$-pseudoephedrine, and changed the diastereomers collision-cross sections. Therefore, each cluster showed different mobility. ${ }^{24}$

Collisions of chiral ions and molecules were used to study the influence of chirality on the ion-molecule complexes formed and their fragmentation pattern and yield. Kulyk et al. (2017) reported the study of $0.1-10.0 \mathrm{eV}$ low-energy collisions of L- and D-protonated enantiomers of phenylalanine, tryptophan, and methionine with $(R)$ - and $(S)$-2-butanol, and $(S)$-1-phenylethanol chiral selectors targets. Phenylethanol was used to intensify the steric interactions with the amino acids compared to those of 2-butanol. They found that ionmolecule complex formation followed by dissociation occurred and was independent of the chirality of targets and ions; the fragments yield was also independent. ${ }^{18}$ With a higher energy collision, equivalent results were obtained. ${ }^{25}$ In a similar investigation, CID of proton-bound adducts of tryptophan and 2-butanol indicated that the heterochiral adduct was more stable in low-energy collisions with argon than the homochiral adduct. ${ }^{26}$ In another study, enantiomers of phenylalanine, tryptophan, and methionine were used to form nonprotonated adducts with enantiomers of 1-phenylethanol. Experimental results showed no influence of chirality on the fragmentation of the adducts upon collisions with Ar. Calculations showed a difference in the Gibbs energies of only $\sim 0.5 \mathrm{Kcal} / \mathrm{mol}$ between the formation of homochiral and heterochiral adducts of Met and Try and no difference for those of Phe. ${ }^{27}$ However, this study did not report statistical data to determine if the observed differences in the Gibbs energies were signicant.

Investigations on spectroscopy and photophysics of clusters of enantiomeric species provide insights into the IMS enantiomer separation with chiral selectors. Zehnacker (2014) conducted studies in spectroscopy and photophysics of chiral molecules or protonated ions, and their weakly bound complexes, isolated in the gas phase in jet-cooled conditions. She concluded that the ancillary interactions responsible for chiral recognition, like $\mathrm{OH} \ldots \pi$ or $\mathrm{CH} \ldots \pi$, would be blurred at room temperature. ${ }^{28}$ Ion mobility experiments at high temperatures would be even more blurred.

Nagy et al. states that, to date, most IMS enantioseparations depend on the formation of chiral non-covalent complexes, where the $\mathrm{D}$ or L analyte ions complex with other chiral molecules using transition metal cations. ${ }^{14}$ When reviewing the seven studies cited, there were no enantiomer separations by only injecting a chiral selector, the objective of our study (Table S1). Therefore, two reports claim IMS enantiomer separation ${ }^{20,21}$ while others show the failure of these experiments ${ }^{22}$ or their unsuitability to single collision conditions $18,25,27$ because in the traditional IMS tube thousands of ion-chiral selector collisions have been found. ${ }^{29}$ Because of the inconsistent results of previous gas-phase IMS enantiomer separations and doubts and difficulties on their implementation, ${ }^{14,22,28}$ in this study, drift-tube ion mobility spectrometry coupled to quadrupole mass spectrometry was used and energy calculations were made to study the viability of the separation of mixtures of enantiomers of chiral $\alpha$-amino acids and drugs.

\section{EXPERIMENTAL SECTION}


Chiral separations of racemic mixtures of enantiomers $\alpha$-amino acids and chiral drugs were evaluated by introducing chiral selectors into the buffer gas of an IMS-MS instrument.

\section{Experiments performed}

Several experiments were performed to study the effect on enantiomer separation. In the experiments, the following conditions of the IMS instrument and sample were changed, including the reasons for the experiments:

- Stability of the mobilities of valine enantiomers with time, to rule out a difference in mobility between the enantiomers due to pressure changes or changes in other instrumental parameters when switching from one enantiomer to another. Experimental conditions are described in the legend to Figure 1.

- Buffer gas temperature: because temperature affects the interaction analyte ion-chiral selector. Experimental conditions are described in the legend to Figure 5

- Analyte concentration. Experimental conditions are described in the legend to Figure 4.

- Chiral selector concentration in the buffer gas: because it changes the extent of the interactions with the ions. Experimental conditions are described in the legend to Figure 2.

- Chiral selector nature: because the strength of the interactions depends on the structure of the chiral selectors. Experimental conditions are described in Section 5, Results and Discussion.

- ESI voltage and the acidity of the ESI solvent: to evaluate the possibility of racemization of enantiomer mixtures due to solvent conditions or drift voltage. Experimental conditions are described in Section 6, Results and Discussion.

- Proportions of $\mathrm{D}$ and $\mathrm{L}$ enantiomers in the racemic mixtures: to identify if racemization of the enantiomer mixture was occurring. Experimental conditions are described in Section 6, Results and Discussion.

Instrumental setup. Experiments were performed using an electrospray-ionization atmospheric-pressure ion mobility spectrometer interfaced through a $40-\mu \mathrm{m}$ pinhole to a quadrupole mass spectrometer (Figure S1) in Washington State University (WA, USA) between 2007-2009. The IMS instrument was built at Washington State University and its full description and schematics can be found elsewhere. ${ }^{30} \mathrm{~A}$ brief description is given here. The instrument was equipped with an electrospray ionization source and a drift tube. The tube consisted of a desolvation and drift regions operating in positive mode and separated by a Bradbury-Nielsen-type ion gate. Typical operating parameters used with this instrument

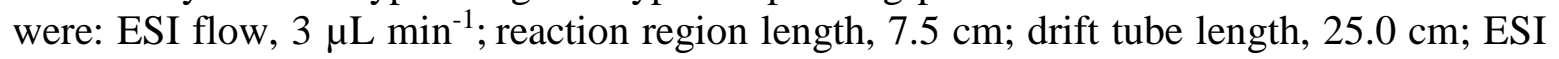
voltage, $15.6 \mathrm{kV}$; voltage at first ring, $12.12 \mathrm{kV}$; voltage at the gate, $10.80 \pm 0.01 \mathrm{kV}$; gate closure potential, $\pm 40 \mathrm{~V}$; gate pulse width, $0.1 \mathrm{~ms}$; scan time, $35 \mathrm{~ms}$; pressure, $680-710$ Torr; buffer gas, nitrogen; buffer gas temperature, $150 \pm 2{ }^{\circ} \mathrm{C}$; buffer gas flow, 1 liter $\mathrm{min}^{-1}$; chiral selector flow rates, 1 to $50 \mu \mathrm{L} \mathrm{hr}^{-1}$.

Materials and reagents. Chirally pure analytes were purchased from Sigma-Aldrich (St. Louis, MO, USA) including alanine, arginine, asparagine, histidine, lysine, methionine, phenylalanine, serine, threonine, tryptophan, tyrosine, valine, valinol, penicillamine, (L)- 
ethyl lactate, methyl (S)-2-chloropropionate, (R)- $\alpha$-(trifluoromethyl) benzyl alcohol (tFMBA), (R)-tetrahydrofuran-2-carbonitrile, R and S-1-phenyl ethanol, atenolol, 2-butanol, and 2,4-dimethylpyridine (2,4-lutidine). These chemicals were ACS reagent grade and their purities $\geq 98 \%$. Water, methanol, and acetic acid were HPLC grade and purchased from J. T. Baker (Phillipsburg, NJ, USA). These chemicals were selected to continue a previous study started in this laboratory that used these chemicals. ${ }^{20}$ They also provided a series of compounds required to test the effect of molecular weight and steric hindrance on chiral separation. The structures of compounds used in this investigation are presented in Figure $\mathrm{S} 2$.

Sample and chiral selector introduction. Liquid samples or blank solution (ESI solution) were electrosprayed at a flow rate of $\sim 3 \mu 1 \mathrm{~min}^{-1}$ into the drift tube. The liquid chiral selectors were injected with gas tight syringes into the buffer gas line, using a T-junction, before the buffer gas heater (Figure S1). To help vaporize the chiral selector, the temperature of the Tjunction was increased to $\sim 150{ }^{\circ} \mathrm{C}$ using a heating tape.

Identification of compounds. Analytes were identified by comparing their $m / z$ signal in mass spectrometry to the molecular weight of their protonated molecules or clusters. Also, reduced mobilities of protonated analytes were compared with those from literature. Additionally, SIM-IMS was performed on the analyte peaks and their clusters to identify the peaks in the IMS spectrum.

Quantitation. Electrospray-ionization ion mobility spectrometry (ESI-IMS) is considered a semi-quantitative technique. The ionization process limits quantitation in ESI-IMS because analytes are ionized by charge transfer reactions. Concentration in the electrosprayed sample cannot be derived from peak height because processes of charge competition can hinder complete ionization of the analyte. Nevertheless, this competition does not affect the expected enantiomeric ratios of the solutions because enantiomers have the same proton affinity and ionization probability.

Instrument calibration. Under certain conditions, the product of an ion reduced mobility, $K_{0}$, times its drift time is constant. This fact allows the reduced mobility of ions to be calculated from that of a calibrant, $K_{0, c}$, the drift time of the calibrant at the specific conditions of the experiment, $\mathrm{c}_{\mathrm{t}}$, and the drift time of the analyte at the same conditions, $\mathrm{d}_{\mathrm{t}}$ :

$$
K_{0}=K_{0, c} \frac{c_{t}}{d_{t}}(1)
$$

2,4-lutidine, commonly selected as calibrant, was used in these experiments. The use of this equation eliminates the necessity of reading barometers and the errors due to mismeasurements of temperature, pressure and voltages required to calculate $K_{0}$.

Computational Studies. The formations of complexes between (S)-2-butanol and protonated (R,S)-atenolol, (R,S)-serine and (R,S)-valinol in the gas phase were investigated computationally. Atenolol was protonated at the nitrogen atom of the isopropylamine group, and serine and valinol at the amine group. 
The molecules in this study are very flexible and have many local energy minima with similar energies. In order to identify representative structures, a search of the conformational space was performed. First, the CREST program, which uses a meta-dynamic driven search algorithm, was employed to find low-energy conformers ${ }^{31}$. The algorithm consists of several molecular dynamic simulations. The structures within an energy window of $9 \mathrm{kcal} / \mathrm{mol}$ were selected for further consideration. Furthermore, to improve the sampling of the conformation space, the simulation times (which are chosen automatically, depending on the flexibility of the system) were increased from their default values (atenolol: 185 ps, butanol: 84 ps, serine: 85 ps, atenolol-butanol complexes: 640 ps, serine-butanol complexes: 202 ps, valinol: 112 ps, valinol-butanol complexes: 290 ps). The semi-empirical tight-binding method GFN2xTB was employed in these calculations, which has been shown to yield reasonable structures and non-covalent interactions ${ }^{32,33}$.

In the next step, all structures obtained in the previous conformation search were reoptimized and vibrational frequencies were calculated using the B97-3c density functional method, which is very efficient computationally, making it possible to optimize the geometries and calculate the vibrational frequencies of hundreds of molecules. This method has been shown to perform well for non-covalent interactions ${ }^{34}$. In the B97-3c method, a triple -zeta basis is applied and the D3 dispersion correction is included. Structures with imaginary frequencies were discarded. Next, the set of remaining structures was searched for duplicate structures, which were eliminated. Duplicate structures were identified based on the RMSD, which was calculated using the DockRMSD program ${ }^{35}$.

The structures with the lowest free energy were selected and single point energy calculations were performed employing the $\omega \mathrm{B} 97 \mathrm{X}-\mathrm{V}$ density functional ${ }^{36}$ in combination with the def2QZVP basis set ${ }^{37,38}$. This basis set was chosen in order to minimize the basis-set superposition error. We also performed analyses using the complete ensembles of structures obtained after B97-3c minimization. However, the results were very similar to those obtained with only the lowest energy structure and, therefore, are not presented here.

All DFT calculations were performed with the ORCA program, version 4.2.1 ${ }^{39-41}$. To speed up the calculations with the $\omega \mathrm{B} 97 \mathrm{X}-\mathrm{V}$ functional, the RIJ-COSX approximation ${ }^{42-45}$ was applied. Thermodynamic quantities were calculated using the geometries and vibrational frequencies obtained at the B97-3c level of theory. Thermodynamic data were calculated at a temperature of $423 \mathrm{~K}$, consistent with the experimental conditions. All figures of the molecules were created with VMD. ${ }^{46}$

\section{Results and Discussions}

Chiral separations of racemic mixtures of $\alpha$-amino acids and chiral drugs were evaluated by introducing chiral selectors into the buffer gas of an ion mobility-quadrupole mass spectrometer. MS spectra (500 averages per spectrum) and mobility spectra (at least 250 averages per spectrum) were acquired after stabilizing the chiral selector concentration for at 
least one hour and when the ratio of the peak height of the protonated molecule to the analytechiral selector cluster peak was reproducible from one spectrum to the next, indicating stable conditions in the drift tube. The reproducibility of the reduced mobilities was $<2 \%$, calculated as the relative standard deviation of the reduced mobilities of five different samples with the same concentration prepared independently and analyzed on different days. The repeatability of the reduced mobilities was $<0.5 \%$, calculated as the relative standard deviation of the reduced mobilities of five or more consecutive analyses (1600 averages each) of the sample.

\section{Stability of the mobilities of valine enantiomers with time}

Figure 1 shows the drift time of the ions of $943-\mu \mathrm{M}$ solutions of valine enantiomers and racemic mixtures of valine when $0.69 \mathrm{mmol} \mathrm{m}^{-3}$ of $(\mathrm{S})$-2-butanol was introduced into the buffer gas in an 8-hr period at $150{ }^{\circ} \mathrm{C}$. For both racemic data points in this experiment, the racemic mixtures were prepared mixing the enantiomer solutions used to obtain the data for this figure. Data were obtained in the SIM-IMS mode selecting the mass of protonated valine. The drift times were always different for both enantiomers in this experiment. The analyses were made by switching between the $\mathrm{D}$ and $\mathrm{L}$ enantiomers of valine to discard that changes in the mobilities were produced by pressure changes when switching from one set of enantiomers to another. There was a minimum drift time separation of $\sim 0.2 \mathrm{~ms}$ between the two enantiomers and a maximum of $\sim 0.45 \mathrm{~ms}$. This separation was large enough to observe the individual peaks of the enantiomers in a mixture, considering that the resolving power of the mobility spectrometer was high $(\sim 100$, Figure S3). However, the racemic mixtures yielded only one peak, with a drift time that matched that of D-valine. The standard deviations of the drift times were $0.08 \mathrm{~ms}$ (L-valine) and $0.05 \mathrm{~ms}$ (D-valine) over the 8-hr period; this variation was caused mainly by the atmospheric pressure drift. In other five analogous experiments, the enantiomers kept similar separations and positions at different (S)-2-butanol flow rates and temperatures on different days. However, in several other experiments with different $\alpha$-amino acids and chiral selectors this drift time difference between D and L could not be reproduced (Table S2 and Figures S4 and S5). 


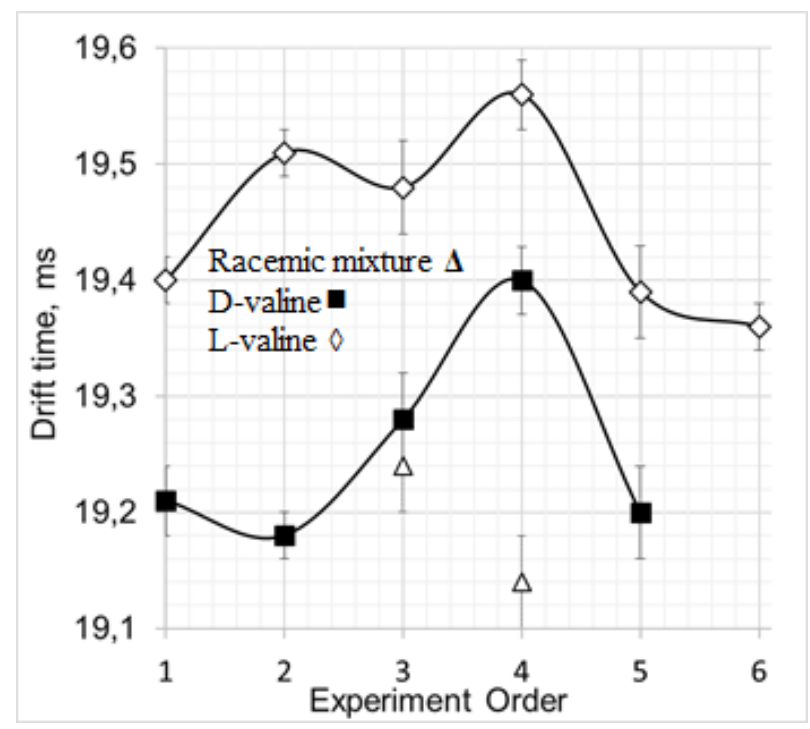

Figure 1. Drift times of valine enantiomers in an 8-hr period using (S)-2-butanol chiral selector. Solutions of D-valine $(\boldsymbol{\square})$, L-valine $(\diamond)$ and racemic mixtures of valine $(\Delta)$ at a $943-$ $\mu \mathrm{M}$ concentration were analyzed when $0.69 \mathrm{mmol} \mathrm{m}^{-3}$ of (S)-2-butanol was introduced into the buffer gas at $150{ }^{\circ} \mathrm{C}$. The drift times were different for both enantiomers although the racemic mixtures yielded only one peak, with a drift time closer to that of D-valine. Table S3 shows the data for this graph. 


\section{Chiral selector concentration and enantiomer separation}

Figure 2 shows that the increase in chiral selector concentration from 0 to $6.8 \mathrm{mmol} \mathrm{m}^{-3}$ in the buffer gas increased the drift times of valinol enantiomers at $125^{\circ} \mathrm{C}$. Although there was always a drift time difference between both enantiomers (an average difference of $0.26 \mathrm{~ms}$ ), only one peak was obtained when the racemic mixture was electrosprayed, as in the experiment described in Figure 1. Figure 2 also shows that the curve at $125{ }^{\circ} \mathrm{C}$ flattened at high (S)-2-butanol concentrations; this flattened curve indicates a ligand saturation of the sites on the valinol ion available for attachment of chiral selector at high concentrations of (S)-2-butanol. At $200^{\circ} \mathrm{C}$, the mobility of valinol ions was unaffected by the increase in chiral selector concentration in the buffer gas because electrostatic interactions valinol-(S)-2butanol decreased probably due to the weak analyte-ligand bonds at high temperature. At 1.3 mmol m${ }^{-3}$ of (S)-2-butanol concentration in the buffer gas, the superimposed IMS spectra of individually-injected valinol enantiomers showed different drift times (Figure S6a) and their racemic mixture only one peak (Figure S6b).

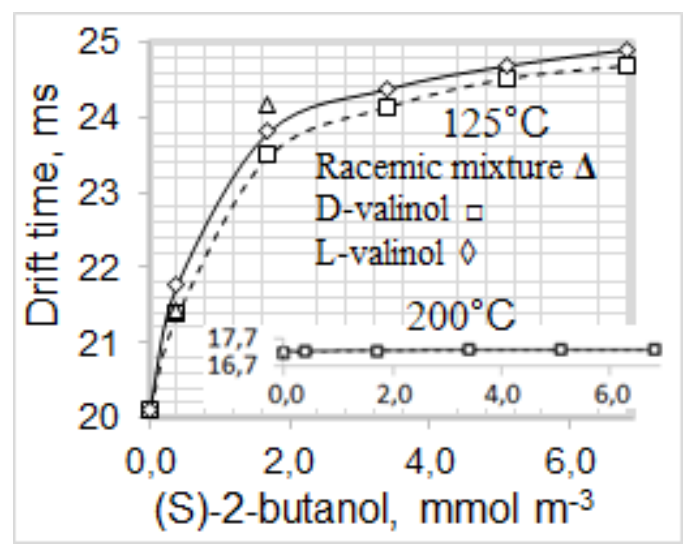

Figure 2. Effect of chiral selector concentration on the mobilities of valinol enantiomers. 943- $\mu \mathrm{M}$ solutions of D-valinol ( $\square$ ), L-valinol $(\diamond)$ and racemic mixtures $(\Delta)$ of valinol were analyzed at a buffer gas temperature of $125^{\circ} \mathrm{C}$ and $200{ }^{\circ} \mathrm{C}$ (inset). At $200^{\circ} \mathrm{C}$, the enantiomers had the same drift time because the interaction valinol-(S)-2-butanol decreased by the weak analyte-ligand bonds at high temperature and the difference in drift time between the enantiomers was too small. The $\mathrm{x}$ axis shows the chiral selector concentration in the buffer gas. Mobilities were measured alternating between the $\mathrm{R}$ and $\mathrm{S}$ enantiomers. Other experimental conditions were as those in Figure 1. See Table S4 for drift time values.

In other experiment with phenylalanine enantiomers, (R)-1-phenyl ethanol was used as chiral selector between 0 and $11 \mathrm{mmol} \mathrm{m}{ }^{-3}$ at $175^{\circ} \mathrm{C}$. Different drift times were obtained for the pure enantiomers when (R)-1-phenyl ethanol was introduced into the buffer gas but no racemic mixtures were tested. Mobilities were measured alternating between the $\mathrm{R}$ and $\mathrm{S}$ species (less than 5 minutes between one enantiomer to the next) for every buffer gas chiral selector flow rate; therefore, the differences in mobilities cannot be due to changes in experimental conditions. Results are summarized in Table S5. Similar results were obtained in experiments with D and L valinol (Table S6). However, in spite of the few promising experiments, numerous negative results in similar experiments were obtained (Table S2). 


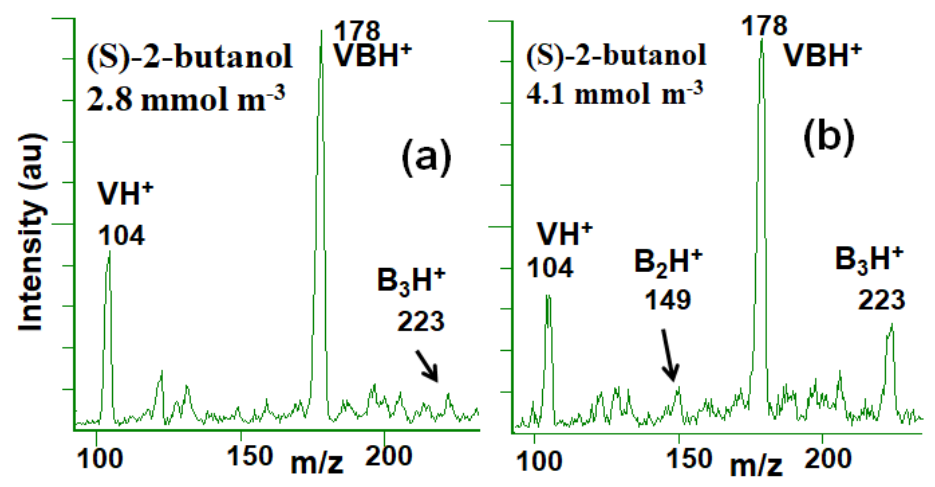

Figure 3. Increasing formation of clusters at high (S)-2-butanol concentrations. MS spectra of a $943-\mu \mathrm{M}$ solution of D-valinol (V) at $150{ }^{\circ} \mathrm{C}$ when the concentrations of (S)-2butanol (B) in the buffer gas increased from (a) $2.8 \mathrm{mmol} \mathrm{m}^{-3}$ to (b) $4.1 \mathrm{mmol} \mathrm{m}^{-3}$. Other experimental conditions were as in Figure 1. When (S)-2-butanol concentration increased, the intensity of $\mathrm{VH}^{+}$decreased with respect to $\mathrm{VBH}^{+}$because the cluster formed from $\mathrm{VH}^{+}$ increased, as expected.

The formation of large valinol-(S)-2-butanol clusters $(\mathrm{m} / \mathrm{z}, 178$, Figure 3) explained the drift time increase of valinol from $\sim 20$ to $\sim 25 \mathrm{~ms}$ (Figure 2) when (S)-2-butanol was introduced into the buffer gas. The intensity of the cluster peak of valinol at $\mathrm{m} / \mathrm{z} 178$ increased with respect to that of the protonated peak of D-valinol at $\mathrm{m} / \mathrm{z} 104$ (Figure 3) when the chiral selector concentration increased from 2.8 to $4.1 \mathrm{mmol} \mathrm{m}^{-3}$, as expected if the cluster was formed from protonated valinol. Figure 3 also illustrates the expected increase in intensity of the peaks of the dimer $\left(\mathrm{B}_{2} \mathrm{H}^{+}, m / z, 149\right)$ and trimer $\left(\mathrm{B}_{3} \mathrm{H}^{+}, m / z 223\right)$ of $(\mathrm{S})$-2-butanol when the chiral selector concentration increased. The unknown peaks at $m / z 133$ and 137 appeared when 2-butanol was used as chiral selector.

\section{Analyte concentration and enantiomer separation}

Figure 4 shows the drift times of solutions of 10, 50, 250, and 943- $\mu \mathrm{M}$ of D- and L-valinol, and racemic mixtures of valinol enantiomers when $0.69 \mathrm{mmol} \mathrm{m}^{-3}$ of (S)-2-butanol was introduced into the buffer gas at $150{ }^{\circ} \mathrm{C}$. The effect of valinol concentration was unimportant on the mobilities of its enantiomers when (S)-2-butanol was introduced into the buffer gas under the conditions of the experiments. As before, the drift times of valinol enantiomers were significantly different $(0.3 \mathrm{~ms}$ average $)$ and the resolving power was high ( 116) when the individual solutions were analyzed, but a racemic mixture only produced a single peak with a drift time similar to that of D-valinol. The standard deviation of the drift times over the length of the experiment was 0.04 (L-valinol) and $0.07 \mathrm{~ms}$ (D-valinol); these variations were caused mainly by changes in atmospheric pressure and, also, in other instrumental parameters. However, individual racemic mixtures of eleven amino acids, glucose, valinol, atenolol and penicillamine at different concentrations were unsuccessfully tested for chiral separation with different concentrations of chiral selectors, including 2-butanol, in the buffer gas at several temperatures and instrumental conditions (Table S2). 


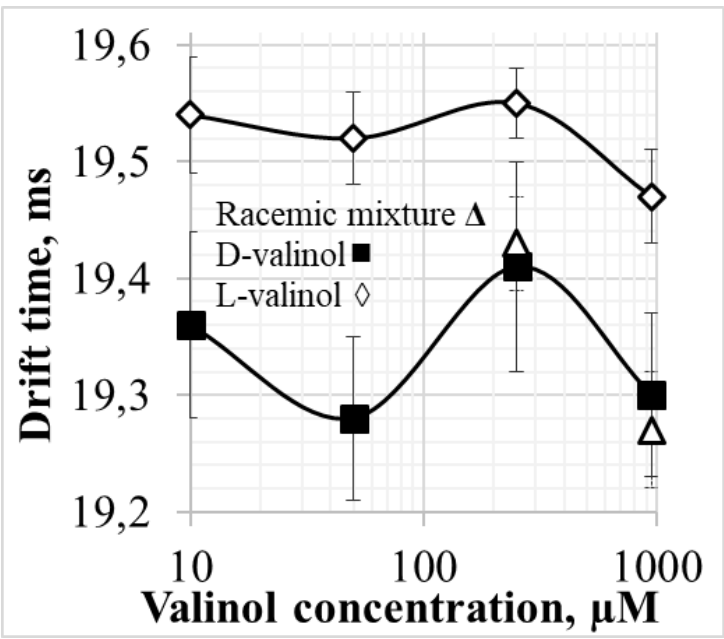

Figure 4. Effect of valinol concentration on the mobilities of its enantiomers. Solutions of D-valinol ( $\boldsymbol{\square})$, L-valinol $(\diamond)$, and racemic mixtures $(\Delta)$ of valinol were analyzed at concentrations of $10,50,250$, and $943-\mu \mathrm{M}$ (logarithmic scale) when $0.75 \mathrm{mmol} \mathrm{m}^{-3}$ of (S)2-butanol was introduced into the buffer gas at $150{ }^{\circ} \mathrm{C}$. The drift times of valinol enantiomers were different $(0.3 \mathrm{~ms}$ in average $)$ but, despite the concentration of the racemic mixture, only a single peak was produced, with a drift time similar to that of D-valinol. Variations in drift time were caused mainly by changes in atmospheric pressure and, also, in other instrumental parameters. Other experimental conditions were like in Figure 1.

\section{Buffer gas temperature and separation of valinol enantiomers}




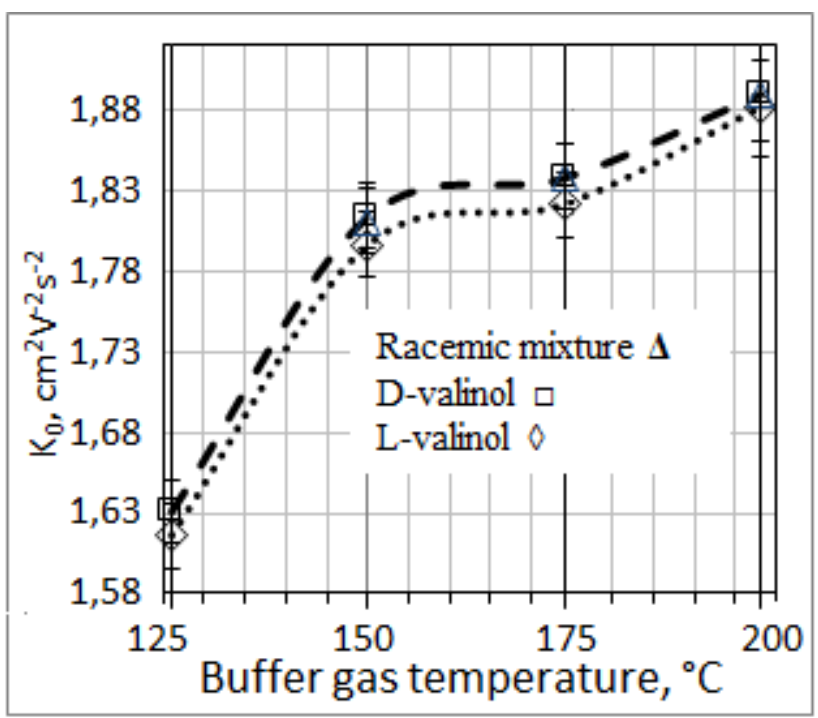

Figure 5. Effect of temperature on the mobilities of valinol enantiomers. $943-\mu \mathrm{M}$ solutions of D-valinol $(\square)$, L-valinol $(\diamond)$, and racemic mixtures of valinol $(\Delta)$ were analyzed when $0.28 \mathrm{mmol} \mathrm{m}^{-3}$ of (S)-2-butanol was introduced into the buffer gas from 125 to $200^{\circ} \mathrm{C}$. The drift time differences between the enantiomers were statistically significant and decreased with increasing temperature (Tables 1 and S7) because the interaction valinol-(S)2-butanol decreased at high temperature. Again, the racemic mixtures yielded only one peak, with a drift time close to that of D-valinol. Other experimental conditions were as in Figure 1.

Figure 5 shows the drift time of the response ions of $943-\mu \mathrm{M}$ solutions of valinol enantiomers and racemic mixtures of valinol when the temperature of the buffer gas was varied from 125 to $200^{\circ} \mathrm{C}$ at a $0.28 \mathrm{mmol} \mathrm{m}^{-3}$ concentration of (S)-2-butanol. The drift times were always different for both enantiomers. The differences in drift times between the L and Denantiomers of valinol were $0.11,0.19,0.17$, and 0.08 at $125,150,175$, and $200{ }^{\circ} \mathrm{C}$, respectively. This decreasing difference as the temperature raised (except for $125^{\circ} \mathrm{C}$ ) indicates that the interaction valinol-(S)-2-butanol decreased probably due to the weak analyte-ligand bonds at high temperature. Again, the racemic mixtures yielded only one peak, with a drift time close to that of D-valinol. This lack of separation was obtained in spite of the fact that the resolving power was high enough $(>100)$ and the drift times of the enantiomers, when individually analyzed, were sufficiently different to obtain a partial separation. Table 1 shows the mobility values and uncertainties for this experiment indicating that the differences in the drift times between the individual enantiomers at the same temperature were significant except for $125^{\circ} \mathrm{C}$ and, therefore, the racemic mixture should have shown separation. Two similar experiments are detailed in Tables S8 and S9. However, these were the exceptions: numerous similar experiments with negative results are compiled in Table S2. 
Table 1. Mobility values and uncertainties for the data in Figure 5. The drift times for the $\mathrm{D}$ and L-enantiomers were significantly different according to the $\mathrm{P}$ values.

\begin{tabular}{|c|c|c|c|c|c|c|c|c|c|c|c|c|c|}
\hline \multirow{2}{*}{\begin{tabular}{|c|}
$\begin{array}{c}\text { Temperature, } \\
{ }^{\circ} \mathrm{C}\end{array}$ \\
\end{tabular}} & \multicolumn{4}{|c|}{ D-valinol } & \multicolumn{4}{|c|}{ L-valinol } & \multirow[t]{2}{*}{$\mathrm{P}^{*}$} & \multicolumn{4}{|c|}{ D/L-valinol } \\
\hline & dt & SD & $\mathrm{n}$ & $\mathrm{K}_{0}$ & dt & SD & $\mathrm{n}$ & $\mathrm{K}_{0}$ & & dt & SD & $\mathrm{n}$ & $\mathrm{K}_{0}$ \\
\hline 125 & 23.35 & 0.07 & 3 & 1.631 & 23.46 & 0.06 & 6 & 1.616 & 0.043 & - & - & & - \\
\hline 150 & 19.23 & 0.03 & 10 & 1.814 & 19.42 & 0.02 & 4 & 1.796 & $<0,0001$ & 19.26 & 0.04 & & 1.811 \\
\hline 175 & 17.98 & 0.02 & 4 & 1.838 & 18.15 & 0.02 & 4 & 1.821 & $<0,0001$ & 17.99 & 0.02 & 6 & 1.838 \\
\hline 200 & 16.50 & 0.00 & 3 & 1.890 & 16.58 & 0.02 & 3 & 1.881 & 0.0023 & 16.50 & 0.01 & 9 & 1.890 \\
\hline
\end{tabular}

dt: drift time; SD: standard deviation. * for the D and L-valinol drift time means. Data was obtained switching between one enantiomer and the other: $\mathrm{L}$, then $\mathrm{D}$, then $\mathrm{L}$ and so on.
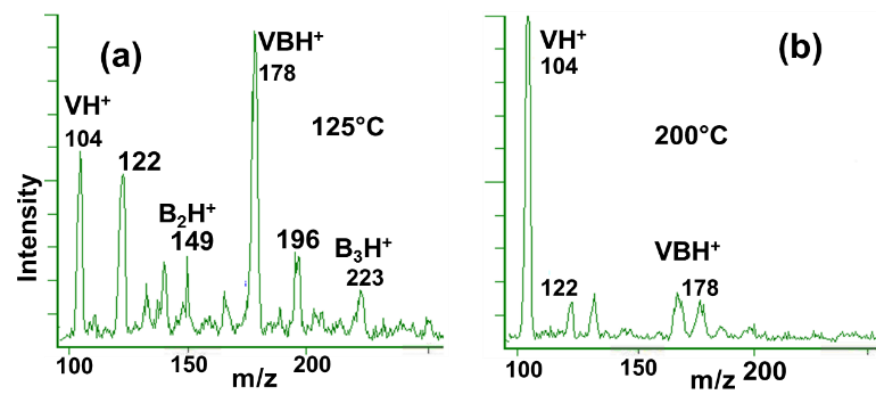

Figure 6. Extensive formation of clusters at low temperatures. (a) MS spectrum of valinol, $\mathrm{V}$, at $125^{\circ} \mathrm{C}$ and (b) at $200{ }^{\circ} \mathrm{C}$. (S)-2-butanol, B, in the buffer gas was $0.28 \mathrm{mmol} \mathrm{m}^{-}$ ${ }^{3}$ and valinol was $943 \mu \mathrm{M}$. A large $\mathrm{VBH}^{+}$peak $(\mathrm{m} / \mathrm{z}, 178)$ and peaks of the dimer $\left(\mathrm{B}_{2} \mathrm{H}^{+}, \mathrm{m} / z\right.$ 149) and trimer $\left(\mathrm{B}_{3} \mathrm{H}^{+}, \mathrm{m} / \mathrm{z}, 223\right)$ of (S)-2-butanol are seen at $125^{\circ} \mathrm{C}$ (Figure 6a). At $200{ }^{\circ} \mathrm{C}$, only a small $\mathrm{VBH}^{+}$peak and a large $\mathrm{VH}^{+}$peak are observed and the clusters $\mathrm{B}_{2} \mathrm{H}^{+}$and $\mathrm{B}_{3} \mathrm{H}^{+}$ disappeared, due to weak electrostatic interactions at high temperatures (Figure 6b). At $\mathrm{m} / \mathrm{z}$ 122 the hydrated valinol ion, $\mathrm{VH}_{3} \mathrm{O}^{+}$, is observed.

The small interaction valinol-(S)-2-butanol at high temperature (Figures 2 and 5) agree with data in the mass spectra in Figure 6. Figure 6a shows a large valinol-(S)-2-butanol cluster peak ( $\mathrm{m} / \mathrm{z}$ 178) and cluster peaks of (S)-2-butanol (at $\mathrm{m} / \mathrm{z} 149$ and 223) at low temperatures $\left(125^{\circ} \mathrm{C}\right)$ but the high temperature spectrum $\left(200{ }^{\circ} \mathrm{C}\right.$, Figure $\left.6 \mathrm{~b}\right)$ only showed a small valinol(S)-2-butanol peak along with a large protonated valinol peak. Additionally, the drift times of valinol remained unchanged with the increase in chiral selector concentration at $200^{\circ} \mathrm{C}$ (Figure 2). This indicates a weak interaction valinol-(S)-2-butanol at high temperature. The (S)-2-butanol dimer $(\mathrm{m} / \mathrm{z}, 149)$ and trimer $(\mathrm{m} / \mathrm{z}, 223)$ did not appear in Figure $6 \mathrm{~b}$ also due to small interactions of (S)-2-butanol with protonated (S)-2-butanol at high temperatures.

\section{Type of chiral selector and separation of enantiomers}

(R) and (S)-1-phenyl ethanol, (R) and (S)-2-butanol, (R)-tetrahydrofuran-2-carbonitrile (tHFCN), and (R)- $\alpha$-(trifluoromethyl) benzyl alcohol were unsuccessfully assayed as chiral selectors for racemic mixtures of the compounds recorded in Table S2 in the specific conditions used. 
Ions of amino acids and valinol were not observed in the spectra when tHFCN was introduced in the buffer gas as a chiral selector at concentrations down to $3.3 \mathrm{mmol} \mathrm{m}^{-3}$. This result can be attributed to charge stripping of tHFCN from valinol ions, and for this, tHFCN would not be a suitable selector for chiral separations in IMS. ${ }^{48}$

\section{Effect of other parameters on the separation of enantiomers}

Other instrumental parameters and conditions were varied to obtain chiral separations. We tested a $50-\mu \mathrm{M}$ racemic mixture of valine with $6.8 \mathrm{mmol} \mathrm{m}^{-3}$ of (R)-2-butanol in the buffer gas as the chiral selector at $175^{\circ} \mathrm{C}$ (unless other conditions are specified) changing a single experimental condition to increase the chiral selector concentration in the buffer gas, to find better conditions for separation, or to rule out an effect of those conditions on a possible racemization or conversion from the $\mathrm{L}$ - to the $\mathrm{D}$-enantiomer. This last objective aims to the results in figures 1 and 4, where the signal for the D-enantiomer overlaps with that of the racemic mixture indicating a possible racemization of the D-enantiomer. In all cases, only individual peaks were obtained for the racemic mixtures:

1. Buffer gas flow rates. Different buffer gas flow rates of 3.6, 1.8, 0.9, and 0.45 L min ${ }^{1}$ were used. These experiments were performed because low buffer gas flow rates increase the chiral selector concentration in the buffer gas and this could increase the possibility of chiral separation. No separation of the racemic mixture was observed in these experiments.

2. ESI voltage. The ESI voltage was varied between 15.6 and $13.9 \mathrm{kV}$; voltage differences of $3.5,2.8,2.4,2.1,1.9$, and $1.8 \mathrm{kV}$ with respect to the target screen were tested. The IMS signal of valine was lost when a voltage difference of $1.8 \mathrm{kV}$ was reached due to the absence of nebulization at those low voltages. These experiments were performed to rule out a racemization induced by the high voltages in the ion source. No separation of the racemic mixture was observed in these experiments.

3. Acidity of the ESI solvent. The following experiments were performed to investigate the effect of acidity of the ESI solvent on the separation of enantiomers. Concentrations of $5 \%, 1 \%, 0.1 \%, 0.03 \%$, and $0.01 \%$ acetic acid in the ESI solution were assayed. The IMS signal of valine was lost below $0.01 \%$ due to insufficient protonation. No separation of the racemic mixture was observed in these experiments.

4. Proportions of $D$ and $L$ enantiomers in the racemic mixture. $D$ and $L$ enantiomers of valine were assayed in 10:90, 30:70, 70:30, and 90:10 proportions (v/v). The reason for these experiments was that the racemic mixture, in some of the experiments detailed in sections 1-4, yielded a drift time similar to that of D-valine and different from that of L-valine. Therefore, it was possible that, for some reason, the Lenantiomer was converting into D when they were mixed. The analyses of mixtures with different proportions of the enantiomers would show a shift from the drift time of $\mathrm{D}$-valine to that of $\mathrm{L}$ when the concentration of $\mathrm{L}$ increased in the mixture, if this conversion was occurring. However, that shift was not observed and no separation of the racemic mixture was observed in these experiments.

5. Buffer gas temperatures. Buffer gas temperatures of $50,80,100$, and $125^{\circ} \mathrm{C}$ were assayed in the analysis of a $943-\mu \mathrm{M}$ racemic mixture of valinol. These temperatures were tested to rule out the possibility of chiral selector racemization upon interaction with the hot metallic surface of the gas heater and to increase the analyte-selector 
interactions. No separation of the racemic mixture was observed in these experiments. This might rule out racemization produced by high temperatures, although lower temperatures should be tried to increase the bonding strength.

\section{Theoretical considerations}

Several possible interactions between chiral 2-butanol and enantiomers of atenolol, serine and valinol cations were studied. These species were selected because they have the highest and lowest molecular weights and the effects of adduction of 2-butanol on their ion mobilities are the smallest and largest, respectively; this is important for the comparisons we will make. In amino acids, the hydrogen bond most energetically favorable was the one produced by the interaction of the hydroxyl group of 2-butanol with the protonated amino group of the analyte but the bond could also occur through the carboxylic group; Figure S7 shows the interaction phenylalanine:2-butanol. Figures of the isolated chiral cations and the complexes are shown in the Supporting Information (Figures S8-S10). In all complexes between butanol and atenolol, serine, and valinol, a hydrogen bond was formed between the protonated amino group of the chiral cation and the $\mathrm{OH}$ group of 2-butanol. In the atenolol complexes a further hydrogen bond was formed between the $\mathrm{OH}$ group of 2-butanol and the oxygen atom of the amide group of atenolol.

The calculated complex formation energies are shown in Table 2. The results obtained with the two functionals and basis sets are very similar and show similar relative stabilities of the complexes formed between 2-butanol and the R- or S-enantiomers of the chiral cations. The formation of all complexes is exothermic. However, the large enthalpy decrease is largely compensated by a loss of entropy, which is largest for atenolol, which also has the smallest binding enthalpy, making the formation of the complex endergonic in this case. The formations of the serine and valinol complexes are all exergonic.

Table 2. Electronic complex formation energies ( $\Delta \mathrm{E}$, electronic energy differences without zero-point vibrational energies), Gibbs free energies $(\Delta \mathrm{G})$, enthalpies $(\Delta H)$ and entropies (reported as $\mathrm{T} \Delta \mathrm{S}$ ) for the complex formation reactions studied, at two different levels of theory. All energies are given in $\mathrm{kJ} / \mathrm{mol}$.

\begin{tabular}{|c|c|c|c|c|c|c|c|}
\hline \multirow{2}{*}{$\begin{array}{l}\text { Reaction of complex } \\
\text { formation }\end{array}$} & \multicolumn{5}{|c|}{ B97-3c } & \multicolumn{2}{|c|}{$\begin{array}{c}\omega B 97 X-V / \text { def2- } \\
\text { QZVP//B97-3c }\end{array}$} \\
\hline & $\Delta \mathbf{E}$ & $\Delta \mathbf{G}$ & $\overline{\Delta \boldsymbol{G}}$ & $\Delta \mathbf{H}$ & $\mathbf{T} \Delta \mathbf{S}$ & $\Delta \mathbf{E}$ & $\Delta \mathbf{G}$ \\
\hline$R-A+S-B \rightarrow R-A \cdot S-B$ & -69.0 & 11.2 & \multirow[b]{2}{*}{11.8} & -62.2 & \multirow{4}{*}{$\begin{array}{l}-73.4 \\
-73.8 \\
-65.3 \\
-65.2 \\
-66.1 \\
-65.6\end{array}$} & & \multirow{4}{*}{$\begin{array}{c}9.0 \\
10.3 \\
-20.5 \\
-22.9 \\
-12.0 \\
-10.0\end{array}$} \\
\hline $\mathrm{S}-\mathrm{A}+\mathrm{S}-\mathrm{B} \rightarrow \mathrm{S}-\mathrm{A} \cdot \mathrm{S}-\mathrm{B}$ & -68.9 & 12.4 & & -61.4 & & $\begin{array}{l}-11.2 \\
-71.0 \\
-92.7\end{array}$ & \\
\hline $\mathrm{R}-\mathrm{Ser}+\mathrm{S}-\mathrm{B} \rightarrow \mathrm{R}-\mathrm{Ser} \cdot \mathrm{S}-\mathrm{B}$ & -94.3 & -22.1 & \multirow{2}{*}{-22.6} & -87.5 & & $\begin{array}{l}-94.9 \\
-85.1\end{array}$ & \\
\hline $\mathrm{S}-\mathrm{Ser}+\mathrm{S}-\mathrm{B} \rightarrow \mathrm{S}-\mathrm{Ser} \cdot \mathrm{S}-\mathrm{B}$ & -95.2 & -23.2 & & -88.4 & & -82.3 & \\
\hline
\end{tabular}




\begin{tabular}{|c|c|c|c|c|c|c|c|}
\hline \multirow{2}{*}{$\begin{array}{l}\text { Reaction of complex } \\
\text { formation }\end{array}$} & \multicolumn{5}{|c|}{ B97-3c } & \multicolumn{2}{|c|}{ 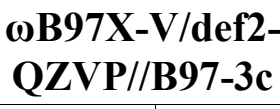 } \\
\hline & $\Delta \mathbf{E}$ & $\Delta \mathbf{G}$ & $\overline{\Delta \boldsymbol{G}}$ & $\Delta \mathbf{H}$ & $\mathbf{T} \Delta \mathbf{S}$ & $\Delta \mathbf{E}$ & $\Delta \mathbf{G}$ \\
\hline $\mathrm{R}-\mathrm{V}+\mathrm{S}-\mathrm{B} \rightarrow \mathrm{R}-\mathrm{V} \cdot \mathrm{S}-\mathrm{B}$ & -86.5 & -13.4 & \multirow{2}{*}{-12.8} & -79.5 & & & \\
\hline $\mathrm{S}-\mathrm{V}+\mathrm{S}-\mathrm{B} \rightarrow \mathrm{S}-\mathrm{V} \cdot \mathrm{S}-\mathrm{B}$ & -84.6 & -12.3 & & -77.9 & & & \\
\hline Complex stabilities & \multicolumn{5}{|c|}{$\Delta \Delta G^{*}$} & \multicolumn{2}{|c|}{$\Delta \Delta \mathrm{G} * *$} \\
\hline Atenolol complexes & \multicolumn{5}{|c|}{1.3} & \multicolumn{2}{|c|}{1.5} \\
\hline Serine complexes & \multicolumn{5}{|c|}{-1.0} & \multicolumn{2}{|c|}{-2.4} \\
\hline Valinol complexes & \multicolumn{5}{|c|}{-1.4} & \multicolumn{2}{|c|}{-2.2} \\
\hline
\end{tabular}

A:atenolol; S-B: (S)-2-butanol; Ser: serine; V: valinol. $\overline{\Delta G}$ : average Gibbs free energy for the complex formation reactions. $\Delta \Delta \mathrm{G}$ : differences of the stabilities of the complexes of the Rand S-enantiomers of the same cation with (S)-2-butanol calculated directly from the complexes (not as differences of the formation free energies) evaluated with B97-3c (*) or $\omega B 97 X-V / d e f 2-Q Z V P / / B 97-3 c\left(^{* *}\right)$. These $\Delta \Delta G$ values differed slightly from those calculated from $\Delta \mathrm{G}$, because the free energies of the isolated R- and S-enantiomers of the chiral cation are not exactly equal in our calculations.

The most important aspect in this investigation is the relative stability of the complexes between chiral butanol and the R- and S-enantiomers of the chiral cation. This stability governs the drift time of the species: the more stable the complex, the longer the species will drift as the large complex, for which it will undergo a more significant number of collisions with the nitrogen drift gas than that of the less stable species and its drift time will be longer. The larger the difference in relative stability of the complexes between chiral butanol and the $\mathrm{R}$ - and S-enantiomers the larger the difference in their drift times and enantiomer separation will be more plausible. It can be seen in Table 2 that $\Delta \Delta \mathrm{G}$ are generally very small, in terms of energies as well as in terms of free energies, with differences in the order of $1-3 \mathrm{~kJ} / \mathrm{mol}$.

\section{Critic review of previous reports on separation of enantiomers}

In 2006, Dwivedi et al. reported the separation of racemic mixtures of amino acids, and other compounds after the introduction of (S)-2-butanol in the buffer gas of a drift-time ion mobility spectrometer, ${ }^{20}$ the same laboratory, instrument, conditions and reagents used in our investigation. We consider those results inconsistent because of the following facts (Figure 7):

a) The curve for (R)-2-butanol must overlap with that of (S)-2-butanol because the adducts L-methionine/(R)-2-butanol and L-methionine/(S)-2-butanol are the specular images of D-methionine/(S)-2-butanol and D-methionine/(R)-2-butanol, respectively, and, therefore, must drift the same time because they have the same size and energetics. 
b) The separation between the curves for L-methionine/(R)-2-butanol and Dmethionine/(R)-2-butanol, and that for D-methionine/(S)-2-butanol and L-methionine/(S)-2butanol must be identical for the same reasoning used in literal a.

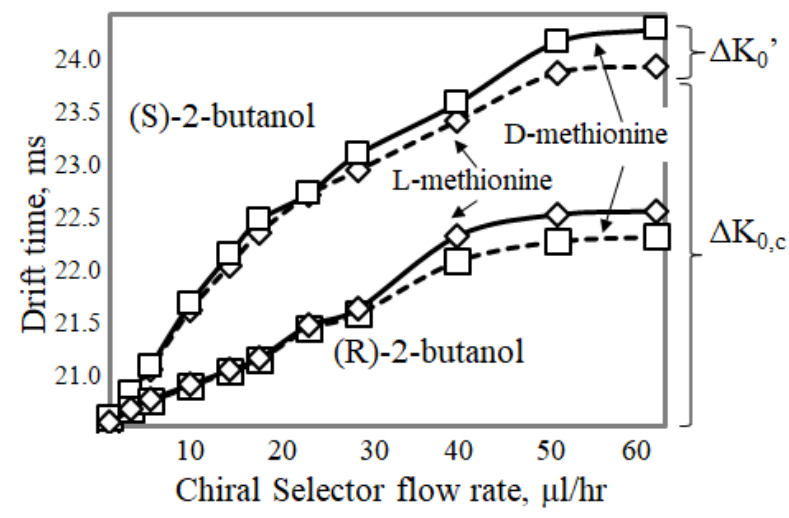

Figure 7. Mobility shifts of methionine enantiomers in the buffer gas in IMS after the introduction of 2-butanol vapor (built from data in Table 1 in reference 20$)^{20} . \Delta K_{0}$ ' and $\Delta \mathrm{K}_{0, \mathrm{c}}$ are shown for racemic methionine and D-methionine, respectively, when (S)-2butanol was injected in the buffer gas.

c) The separation between the enantiomers of atenolol, $\mathbf{\Delta K}_{\mathbf{0}}$ ', was too large, $1.7 \%$ (Table 3 , in bold), compared with those of the other racemic mixtures; it was larger than those of smaller molecules such as tryptophan $(1.6 \%)$, methionine $(0.8 \%)$, glucose $(1.6 \%)$, and penicillamine (1.4\%) in Table S9, and valinol (1.2\%, Table 3), in italics. With a larger size, atenolol should have yielded a $\Delta K_{0}$ ' smaller than those of the previously mentioned species, because a large size decreases the effect of buffer additives on the mobilities. ${ }^{29,48,49}$ Atenolol is a large compound that has shown only a small absolute mobility shift of $0.7 \%$ with 2butanol in the buffer gas for strong non-chiral interactions. ${ }^{50}$ For this reason, it is not clear how a large $1.7 \%$ mobility shift, $\Delta \mathrm{K}_{0}$ ', can be obtained for a weak chiral interaction especially because its non-chiral interaction, stronger than a chiral interaction, for the (S)-atenolol enantiomer $\left(\Delta \mathrm{K}_{0, \mathrm{c}}\right)$ yielded $0 \%$ mobility shift, it did not shift, in close agreement with our non-chiral experiments. ${ }^{29,48-50}$ As a confirmation of this observation, the formation of the atenolol complex resulted endergonic with an average $\Delta \mathrm{G}$ of formation of $11.8 \mathrm{~kJ} / \mathrm{mol}$ (Table 2 ), for which its enantiomers should have not shown any mobility shift with the introduction of 2-butanol in the buffer gas. 
d) The separation between the $D$ - and L-enantiomers of the same species, $\Delta K_{0}$ ', was too large compared to $\Delta \mathbf{K}_{\mathbf{0}, \mathbf{c}}$ (Table 3 , Figure $\left.\mathrm{S} 11\right)$. The $\Delta \Delta \mathrm{G}$ values calculated from reference 20 were small (Table 2 ) as expected from chiral interactions: only $1.3 \mathrm{~kJ} / \mathrm{mol}(0.31 \mathrm{kcal} / \mathrm{mol})$, $-1.0 \mathrm{~kJ} / \mathrm{mol}(0.24 \mathrm{kcal} / \mathrm{mol})$ and $1.4 \mathrm{~kJ} / \mathrm{mol}(0.33 \mathrm{kcal} / \mathrm{mol})$ for atenolol, serine and valinol, respectively, ${ }^{20}$ too small to account for the relatively large enantiomer separations reported. To visualize this, we have to compare the ratio of $\Delta \mathrm{K}_{0}$ ' and $\Delta K_{0, c}$ with respect to $\Delta \Delta \mathrm{G}$ and $\overline{\Delta G}$, respectively (Table 3, last two columns). $\frac{\Delta K_{0, c}}{\overline{\Delta G}}$ and $\frac{\Delta K_{0}{ }^{\prime}}{\overline{\Delta \Delta G}}$ are a measure of the mobility shifts caused by the complex bonding strength and chiral interaction, respectively. For valinol and serine, taken as examples, $\frac{\Delta K_{0}{ }^{\prime}}{\overline{\Delta \Delta G}}>\frac{\Delta K_{0, c}}{\overline{\Delta G}}$; this means that the relative mobilities produced by the chiral interactions in these complexes would be larger than those produced by nonchiral interactions. These results would be contrary to expectations because chiral interactions in these complexes are weaker than their strong non-chiral interactions, including the interaction with the formal charge on the cation. A graphic explanation of this reasoning appears in Figure S11.

Table 3. $\Delta K_{0}$ ' and $\Delta K_{0, c}$ for the data from reference $20 . \Delta K_{0}$ ': percentage mobility difference between enantiomers of the same cation in (S)-2-butanol-doped buffer gas. $\Delta \mathrm{K}_{0, \mathrm{c}}$ : percentage change in $K_{0}$ when butanol flow rate was varied from 0 to $65 \mu \mathrm{L} / \mathrm{hr}$ into the buffer gas. The complete data set of these experiments is shown in Table S9. ${ }^{20}$

\begin{tabular}{|c|c|c|c|c|c|c|c|c|c|c|c|}
\hline \multirow[b]{2}{*}{ Mass } & \multirow[b]{2}{*}{ Species } & \multirow{2}{*}{$\begin{array}{c}\mathrm{K}_{0} \\
\text { value } \\
\text { in } \mathrm{N}_{2}\end{array}$} & \multicolumn{2}{|c|}{$\begin{array}{c}\mathrm{K}_{0} \text { in }(\mathrm{S})-2- \\
\text { butanol }\end{array}$} & \multirow{2}{*}{$\begin{array}{c}\Delta \mathrm{K}_{0}{ }^{\prime} \\
\%\end{array}$} & \multicolumn{2}{|c|}{$\begin{array}{c}\Delta \mathrm{K}_{0, \mathrm{c}} \\
\%\end{array}$} & \multirow[b]{2}{*}{$\overline{\Delta \Delta G}$} & \multirow{2}{*}{$\overline{\Delta G}$} & \multirow{2}{*}{$\frac{\Delta K_{0, c}}{\overline{\Delta G}}$} & \multirow{2}{*}{$\frac{\Delta K_{0}^{\prime}}{\overline{\Delta \Delta G}}$} \\
\hline & & & $\begin{array}{l}\mathrm{D}- \\
\mathrm{ee}^{\#}\end{array}$ & $\begin{array}{l}\text { L- } \\
\text { ee* }\end{array}$ & & D-ee & L-ee & & & & \\
\hline 267 & Atenolol & 1.18 & 1.18 & 1.16 & 1.7 & $0.0^{\&}$ & $-1.7 *$ & $\#$ & \# & \# & \# \\
\hline 104 & Valinol & 1.74 & 1.62 & 1.60 & -1.2 & -6.9 & -8.0 & -1.8 & -12.8 & $0.54^{\ddagger}$ & 0.69 \\
\hline 106 & Serine & 1.73 & 1.55 & 1.52 & -1.9 & -10.4 & -12.1 & -1.7 & -22.6 & $0.46^{¥}$ & 1.14 \\
\hline
\end{tabular}

ee: Enantiomer. ${ }^{\&}$ R-atenolol; *S-atenolol. $\overline{\Delta \Delta G}$ : average difference of the complexes stabilities of the R-and Senantiomers of the same cation with (S)-2-butanol, Table 2. $\overline{\Delta G}$ : average Gibbs free energy for the complex formation reactions. For example: the value 1.2 for the $\Delta \mathrm{K}_{0}$ ' of valinol was calculated as [(1.60-1.62)/1.62]*100 and the value -8.0 for the $\Delta \mathrm{K}_{0, \mathrm{c}}$ for L-valinol was calculated as [(1.60-1.74)/1.74]*100. Mass: mass of the protonated species. \# For atenolol these values would be meaningless because atenolol formation was endergonic. ${ }^{¥}$ the $\Delta \mathrm{K}_{0, \mathrm{c}}$ value for the D-enantiomer was used. A graphic explanation of this table appears in Figure S11.

e) The temperature of the drift region, desolvation region, and drift gas was maintained at $200{ }^{\circ} \mathbf{C}$. At this relatively high temperature, there is a lower effect of 2-butanol on the drift times of the analytes, Figure 2 and literature reports, ${ }^{48,49}$ because at high temperature the bonds are easier to break and the change in drift time should be smaller given that the ions travel most of the time as the non-complexed species. This means that the differences described in literals $\mathrm{c}$ and $\mathrm{d}$ would be larger because our experiments were performed mostly at $150^{\circ} \mathrm{C}$. Also, Zehnacker (2014) concluded that the supplementary interactions accounting for chiral recognition in spectroscopy and photophysics studies of chiral species and their weakly bound complexes should be performed at low temperatures because the interactions would be obscured at room temperature. ${ }^{28}$ For this reason, ion mobility experiments at high temperatures would be even more difficult. 
f) The mass mobility correlation was poor. The correlation between the analyte masses and their ion mobility has been been recognized long ago. ${ }^{51}$ However, correlation coeficients of only 0.24 and 0.45 were obtained from reference 20 for the mass and $\Delta \mathrm{K}_{0}{ }^{\prime}$ and $\Delta \mathrm{K}_{0, \mathrm{c}}$ correlations, respectively (Table S9). Using similar compounds (Phenylalanine, methionine, serine, tryptophan, tyrosine, threonine, ethanolamine, tribenzylamine, tributylamine, the drugs valinol, atenolol, and penicillamine) belonging to several different series of chemical species, we obtained a regression coefficient of 0.74 for valinol, serine, threonine, methionine, phenylalanine, tyrosine, and tryptophan using 2-butanol as a "shift reagent", ${ }^{48}$ the same one used as chiral selector in reference $20 .^{20}$ Those small values of correlation coefficients may indicate that the data supporting the chiral separations reported may not be reliable.

Regarding the separation of enantiomers of the amino compound fluoxetine (Figure S2) using chiral 2-butanol reported by Karas in a patent, ${ }^{21}$ it is not clear how such a large molecule $(309.3 \mathrm{~g} / \mathrm{mol})$ can yield such a large separation and drift. The patent states that (S)-2-butanol was injected in the buffer gas $\left(\mathrm{N}_{2}\right)$. We have studied atenolol, a smaller molecule with 266.3 $\mathrm{g} / \mathrm{mol}$, also an amino compound, injecting 2-butanol in the buffer gas and a saturation of the attaching sites with alcohol molecules on this analyte has been reached before $6.8 \mathrm{mmol} \mathrm{m}^{-3}$ of 2-butanol. This saturation expresses as a stabilization of the mobility of atenolol with higher concentrations of 2-butanol because no additional molecules of 2-butanol can attach to atenolol. This attachment is what produces the mobility shifts in IMS. For atenolol, we have obtained a $\Delta K_{0, c}$ (percentage change in $K_{0}$ when the dopant concentration was varied) of only $-0.7 \%$. From the data in the patent, ${ }^{21} 16.2 \mathrm{~ms}$ for fluoxetine in pure nitrogen buffer gas and $19.52 \mathrm{~ms}$ after adding 2-butanol at $5 \mathrm{~mol} \%$ (molar fraction), a $\Delta K_{0, c}$ of $20.7 \%$ can be calculated with a molecule larger than atenolol. With a larger size, fluoxetine should yield a $\Delta K_{0, c}$ smaller than that of atenolol, $-0.7 \%$, because size decreases the effect of buffer additives on mobilities. ${ }^{29}$ In support of this discussion, even in reference 20 it was found a $\Delta K_{0, c}$ of $0.0 \%$ for the (R)-atenolol enantiomer, as expected. ${ }^{20}$ However, as all patents, it is not peerreviewed and, therefore, its claims have not been verified.

\section{Recommendations for potential chiral separations.}

- Chiral separations in IMS might be obtained with small chiral analytes and large chiral selectors. The mobility of small analytes is more affected by chiral interactions than that of large analytes and large selectors decrease the mobility of ions more than small ones. 49

- The type of chiral interactions between selectors and analytes must be strong, of the polar type, so that the difference between two-points and three-points interactions is large.

- The buffer gas temperature should be low enough to allow these interactions (see text to figures 5 and 6). Figure 5 shows that buffer gas temperature should be around $150^{\circ} \mathrm{C}$ or lower for chiral separation of valinol enantiomers with (S)-2-butanol due to the strong valinol-(S)-2-butanol interactions at low temperatures but this temperature would depend on the nature of analyte and chiral selector. This has to do with the formation of clusters, which slow down the ions and were absent at high temperatures (Figure 6). If separation of enantiomers of amino acids is not obtained before the flattening of the curve of chiral selector concentration vs. mobilities of enantiomers (Figure 2), it is unlikely that 
separation will be obtained at larger chiral selector flow rates because the saturation of the ion with the selector molecules will prevent the chiral interaction.

$\circ$ Water and contamination must be kept out of the drift tube. They cluster with the analyte deforming the chiral sites and obscuring chiral interactions.

\section{CONCLUSIONS}

In contrast to previous studies, ${ }^{20,21}$ no chiral separation was obtained when racemic mixtures of chiral compounds were assayed for separation by introducing chiral selectors into the buffer gas of a drift tube ion mobility-mass spectrometer. After fourteen years of the only published experiment that claimed chiral separation ${ }^{20}$ there have not been reports on separations of enantiomers by injecting volatile chiral selectors in the buffer gas in drift-time IMS. Additionally, several attempts to reproduce those experiments have yielded inconclusive results most of them using the same reagents, instrument, and conditions of the original study. ${ }^{20}$

The lack of separation of the racemic mixtures might have occurred because we did not reproduce the precise conditions used in the previous study. However, chiral separations seemed possible as suggested by the difference in drift times between the enantiomers used in the racemic mixtures when they were individually analyzed. Several experimental conditions and buffer gas selectors were studied for separation of the racemic mixture without success. The lack of enantiomer separation cannot find a plausible explanation: an improper preparation of the racemic mixture cannot be argued because this mixture was prepared combining the same pure enantiomeric solutions, which were yielding different drift times and preferential ionization cannot be hypothesized because enantiomers have the same proton affinity and ionization probability.

The lack of separation of the racemic mixtures was not produced by the conversion of the Lenantiomer into the D-enantiomer in a racemic mixture because in symmetric environments as the ESI solution both enantiomers have the same stability. Acid racemization has been widely demonstrated in amino acids ${ }^{52}$ and could happen due to the acidic conditions of the ESI solution used as a solvent. Bada found that the logarithms of the rate constants of the racemization reactions were about -11 to $25^{\circ} \mathrm{C}$ at $\mathrm{pH} 2$, similar to that of the ESI solvent used in this study. ${ }^{53}$ These speeds are too low to be significant; however, the conditions in our experiments were different: $150^{\circ} \mathrm{C}$, gas phase, and an electric field of $432 \mathrm{~V}$ in the drift tube. If this racemization would have occurred upon interaction with the chiral selector, a different drift time would have been obtained when testing mixtures in different proportions of the two enantiomers or peak broadening would have occurred. One last possible explanation, is that one of the enantiomers has a stronger proton affinity and keeps all the available protons but, again, protons are symmetric and enantiomers must have the same proton affinity.

Finally, energy calculations of the chiral selector -ion interactions showed that these separations are unlikely. Future work must be focused to help obtain reproducible IMS parameters (such as temperature, pressure, and others that affect the mobility of the enantiomers) and to understand the chemistry underlying the singular behavior of the racemic mixture into a drift tube in the presence of a chiral selector. 


\section{ACKNOWLEDGEMENTS}

The corresponding author thanks the kind help of Herbert Hill and Bill Siems in Washington State University for the discussion of Figure 7, central to this paper. This article is dedicated to them. Financial support was provided by a Fulbright scholarship, Universidad de Cartagena, Washington State University and Herbert Hill lab.

The authors declare that they have no known competing financial interests or personal relationships that could have appeared to influence the work reported in this paper.

Doerr is responsible of the theoretical studies and its discussion and Fernandez is responsible of the rest of the paper.

\section{SUPPORTING INFORMATION}

Photograph of the ESI-IMS-MS spectrometer. Structure of reagents. IMS spectrum showing the resolution of the instrument. IMS spectra of racemic mixtures or enantiomers of valinol or atenolol with (S)-2-butanol in the buffer gas. Superimposed IMS spectra of valinol enantiomers and its racemic mixture with (S)-2-butanol in the buffer gas. Drawing of the Interaction phenylalanine:2-butanol. Structures of the isolated chiral cations of the analytes and their complexes with 2-butanol. Graphic explanation of Table 3.

\section{REFERENCES}

1. Tverdislov, V. A.; Yakovenko, L. V. Moscow University Physics Bulletin, 2008, 63, 3, 151-163.

2. Sundaresan, V., \& Abrol, R. (2002). Towards a general model for protein-substrate stereoselectivity. Protein science, 11(6), 1330-1339..

3. W.H. Pirkle, T.C. Pochapsky. Considerations of chiral recognition relevant to the liquid chromatography separation of enantiomers. Chem. Rev. 1989, 89, 347.

4. Schalley, C. A.; Hoernschemeyer, J.; Li, X.; Silva, G.; Weis, P. Distinguishing the topology of macrocyclic compounds and catenanes Int. J. Mass Spectrom. 2003, 228, 2-3, 373-388.

5. Davankov, V. A. (1997). The nature of chiral recognition: Is it a three-point interaction?. Chirality, 9(2), 99-102.

6. Ariëns EJ (1984). Stereochemistry, a basis for sophisticated nonsense in pharmacokinetics and clinical pharmacology. European Journal of Clinical Pharmacology. 26 (6): 663-8. doi:10.1007/bf00541922

7. Woollam, D. H. M. (1962). Thalidomide disaster considered as an experiment in mammalian teratology. British medical journal, 2(5299), 236. 
8 Li, Z., Hu, C., Liu, Y., Li, Q., Fu, Y., \& Chen, Z. (2021). Facile preparation of ethanediamine- $\beta$-cyclodextrin modified capillary column for electrochromatographic enantioseparation of Dansyl amino acids. Journal of Chromatography A, 462082.

9 Zeid, A. M., Nasr, J. J. M., Belal, F., Walash, M., Kaji, N., \& Baba, Y. (2021). Microfluidic fast chiral separation of baclofen and phenylalanine enantiomers based on cyclodextrinelectrokinetic chromatography. Microchemical Journal, 160, 105770.

10. Gübitz, G., \& Schmid, M. G. (2007). Advances in chiral separation using capillary electromigration techniques. Electrophoresis, 28(1-2), 114-126.

11. Zhou, L., Lu, Y., \& Sun, G. (2021). Open tubular capillary column immobilized with sulfobutylether- $\beta$-cyclodextrin for chiral separation in capillary electrochromatography. Journal of Separation Science. In press.

12. Zhang, Q., Ren, S., Xue, S., Li, A., Liu, S., \& Sun, X. (2021). Tetraalkylammonium-1tartrate ionic liquids as sole chiral selectors in capillary electrophoresis. Separation and Purification Technology, 256, 117842.

13. J. Diana Zhang, K.M. Mohibul Kabir, W. Alexander Donald, Chapter Three - IonMobility Mass Spectrometry for Chiral Analysis of Small Molecules, Editor(s): W. Alexander Donald, James S. Prell, Comprehensive Analytical Chemistry, Elsevier, Volume 83, 2019, Pages 51-81, ISSN 0166-526X, ISBN 9780444641540, https://doi.org/10.1016/bs.coac.2018.08.009.

14. Nagy, G., Chouinard, C. D., Attah, I. K., Webb, I. K., Garimella, S. V., Ibrahim, Y. M., ... \& Smith, R. D. (2018). Distinguishing enantiomeric amino acids with chiral cyclodextrin adducts and structures for lossless ion manipulations. Electrophoresis, 39(24), 3148-3155.

15. Sawada, M. (1997). Chiral recognition detected by fast atom bombardment mass spectrometry. Mass spectrometry reviews, 16(2), 73-90.

16. Tao, W. A., \& Cooks, R. G. (2003). Peer reviewed: chiral analysis by MS. Analytical chemistry, 75(1), 25-A.

17. Wu, Q., Wang, J. Y., Han, D. Q., \& Yao, Z. P. (2020). Recent advances in differentiation of isomers by ion mobility mass spectrometry. TrAC Trends in Analytical Chemistry, 124, 115801.

18. K. Kulyk, O. Rebrov, M. Ryding, R.D. Thomas, E. Uggerud, M. Larsson. Low-Energy Collisions of Protonated Enantiopure Amino Acids with Chiral Target Gases. J. Am. Chem. Soc. Mass Spectrom. 2017, 28, 2686.

19. Rister, A. L., \& Dodds, E. D. (2020). Steroid analysis by ion mobility spectrometry. Steroids, 153, 108531. 
20. Dwivedi, P.; Wu, C.; Matz, L. M.; Clowers, B. H.; Siems, W. F.; Hill, H. H. Jr. Gasphase chiral separations by ion mobility spectrometry. Anal. Chem. 2006, 78, 24, 8200-8206.

21. M. Karas. Separation of components of an analysis sample in an ion mobility spectrometer using a supply of selectively interactive gaseous particles. U.S. Patent No. 7015462B2. 21 Mar. 2006.

22. Roscioli, K. M. (2012). Selective ionization and separation in ion mobility spectrometry. Ph.D. Thesis. DOI: 10.6084/m9.figshare.14697012

23. Pérez-Míguez, R. et al. (2019). Chiral discrimination of DL-amino acids by trapped ion mobility spectrometry after derivatization with (+)-1-(9-fluorenyl) ethyl chloroformate. Analytical chemistry, 91(5), 3277-3285.

24. Holness, H.K., Jamal, A., Mebel, A., Almirall, J.R.: Separation mechanism of chiral impurities, ephedrine and pseudoephedrine, found in amphetamine-type substances using achiral modifiers in the gas phase. Anal. Bioanal. Chem. 404, 2407-2416 (2012)

25. K. Kulyk, O. Rebrov, M.H. Stockett, J.D. Alexander, H. Zettergren, H.T. Schmidt, et al. High-energy collisions of protonated enantiopure amino acids with a chiral target gas. Int. J. Mass Spectrom. 2015, 388, 59.

26. O. Rebrov, K. Kulyk, M. Ryding, R. Thomas, E. Uggerud, M. Larsson. Chirally sensitive collision induced dissociation of proton-bound diastereomeric complexes of tryptophan and 2-butanol. Chirality. 2017, 29, 115.

27. O. Rebrov; M. Poline; M. Mauritz; R. Thomas; E. Uggerud; M. Larsson. Non-covalently Bonded Diastereomeric Adducts of Amino Acids and (S)-1-Phenylethanol in Low-energy Dissociative Collisions. Mol. Phys. 2020, 118(4), 1615145.

28. Zehnacker, A. (2014). Chirality effects in gas-phase spectroscopy and photophysics of molecular and ionic complexes: contribution of low and room temperature studies. International Reviews in Physical Chemistry, 33(2), 151-207.

29. Fernandez-Maestre R. Buffer gas additives (modifiers/shift reagents) in ion mobility spectrometry: Applications, predictions of mobility shifts, and influence of interaction energy and structure. J Mass Spectrom. 2018 53(7) 598-613. https://doi.org/10.1002/jms.4190.

30. Wu, C., Siems, W. F., Asbury, G. R., \& Hill, H. H. (1998). Electrospray ionization highresolution ion mobility spectrometry- mass spectrometry. Analytical chemistry, 70(23), 4929-4938.

31. Pracht, P., Bohle, F. \& Grimme, S. Automated exploration of the low-energy chemical space with fast quantum chemical methods. Phys. Chem. Chem. Phys. (2020) doi:10.1039/C9CP06869D.

32. Bannwarth, C., Ehlert, S. \& Grimme, S. GFN2-xTB-An Accurate and Broadly 
Parametrized Self-Consistent Tight-Binding Quantum Chemical Method with Multipole Electrostatics and Density-Dependent Dispersion Contributions. J. Chem. Theory Comput. 15, 1652-1671 (2019).

33. Bannwarth, C. et al. Extended tight-binding quantum chemistry methods. WIREs Comput Mol Sci (2020) doi:10.1002/wcms.1493.

34. Brandenburg, J. G., Bannwarth, C., Hansen, A. \& Grimme, S. B97-3c: A revised low-cost variant of the B97-D density functional method. J. Chem. Phys. 148, 064104 (2018).

35. Bell, E. W. \& Zhang, Y. DockRMSD: an open-source tool for atom mapping and RMSD calculation of symmetric molecules through graph isomorphism. J Cheminform 11, 40 (2019).

36. Mardirossian, N. \& Head-Gordon, M. $\omega$ B97X-V: A 10-parameter, range-separated hybrid, generalized gradient approximation density functional with nonlocal correlation, designed by a survival-of-the-fittest strategy. Phys. Chem. Chem. Phys. 16, 9904-9924 (2014).

37. Weigend, F. \& Ahlrichs, R. Balanced basis sets of split valence, triple zeta valence and quadruple zeta valence quality for $\mathrm{H}$ to $\mathrm{Rn}$ : Design and assessment of accuracy. Phys. Chem. Chem. Phys. 7, 3297-3305 (2005).

38. Weigend, F. Accurate Coulomb-fitting basis sets for $\mathrm{H}$ to $\mathrm{Rn}$. Phys. Chem. Chem. Phys. 8, 1057-1065 (2006).

39. Neese, F. The ORCA program system. WIREs Comput. Mol. Sci. 2, 73-78 (2012).

40. Neese, F. Software update: the ORCA program system, version 4.0. WIREs Computational Molecular Science 8, e1327 (2018).

41. Neese, F., Wennmohs, F., Becker, U. \& Riplinger, C. The ORCA quantum chemistry program package. J. Chem. Phys. 152, 224108 (2020).

42. Kossmann, S. \& Neese, F. Comparison of two efficient approximate Hartee-Fock approaches. Chemical Physics Letters 481, 240-243 (2009).

43. Neese, F., Wennmohs, F., Hansen, A. \& Becker, U. Efficient, approximate and parallel Hartree-Fock and hybrid DFT calculations. A 'chain-of-spheres' algorithm for the Hartree-Fock exchange. Chem. Phys. 356, 98-109 (2009).

44. Izsák, R. \& Neese, F. An overlap fitted chain of spheres exchange method. J. Chem. Phys. 135, 144105 (2011).

45. Neese, F. An improvement of the resolution of the identity approximation for the formation of the Coulomb matrix. J. Comput. Chem. 24, 1740-1747 (2003). 
46. Humphrey, W., Dalke, A. \& Schulten, K. VMD: Visual molecular dynamics. J. Mol. Graph. 14, 33-38 (1996).

48. Fernandez-Maestre R, Wu C, Hill HH. Using a Buffer Gas Modifier to Change Separation Selectivity in Ion Mobility Spectrometry, Int J Mass Spectrom 2010, 298, 2-9. https://doi.org/10.1016/j.ijms.2010.08.009

49. Fernandez-Maestre R, Wu C, Hill HH. Buffer gas modifiers effect resolution in ion mobility spectrometry through selective ion-molecule clustering reactions. Rapid Commun Mass Spectrom 2012, 26, 19, 2211-2223.

50. Fernandez-Maestre, R.; Harden, C.S.; Ewing, R.G.; Crawford, C.L.; Hill Jr., H.H. Chemical Standards in Ion Mobility Spectrometry. Analyst, 2010, 135(6):1433-1442. https://doi.org/10.1039/b915202d

51. Karasek, F. W., \& Kane, D. M. (1972). Plasma chromatography of the n-alkyl alcohols. Journal of Chromatographic Science, 10(11), 673-677.

52. Kreil, G. (1994). Conversion of L-to D-amino acids: a posttranslational reaction. Science, 266(5187), 996-998.

53. Bada, J. L. (1972). Kinetics of racemization of amino acids as a function of pH. Journal of the American Chemical Society, 94(4), 1371-1373. 


\section{On the Separation of Enantiomers by Drift Tube Ion Mobility Spectrometry}

Roberto Fernández-Maestre, ${ }^{1,2}$ and Markus Doerr ${ }^{3}$

\section{Supporting Information}

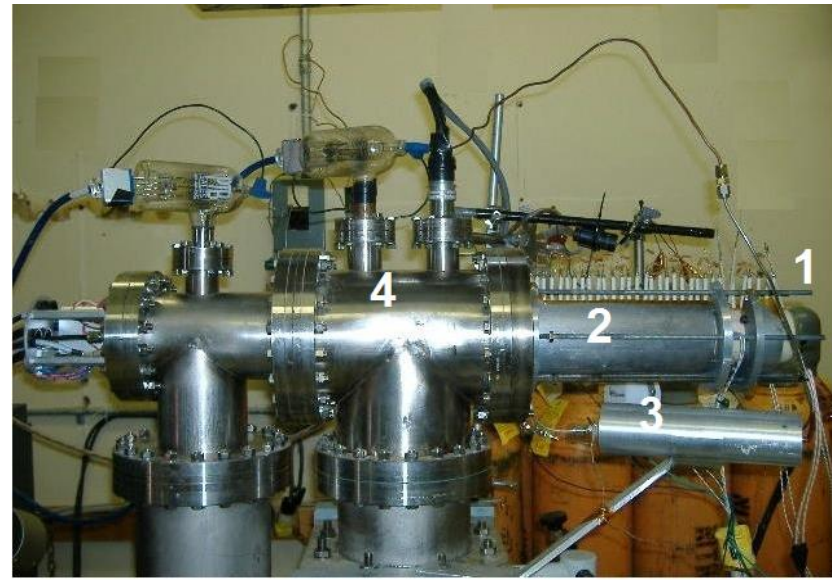

Figure S1. Photograph of the electrospray ionization-atmospheric pressure ion mobility-mass spectrometer. 1. Desolvation region 2. Drift region 3. Buffer gas heater 4. Mass spectrometer 

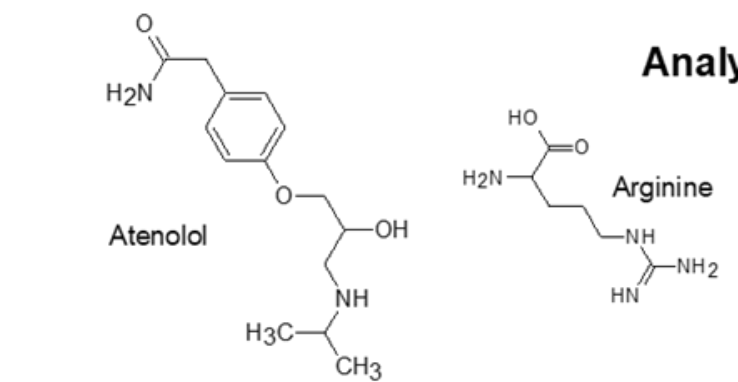

Analytes
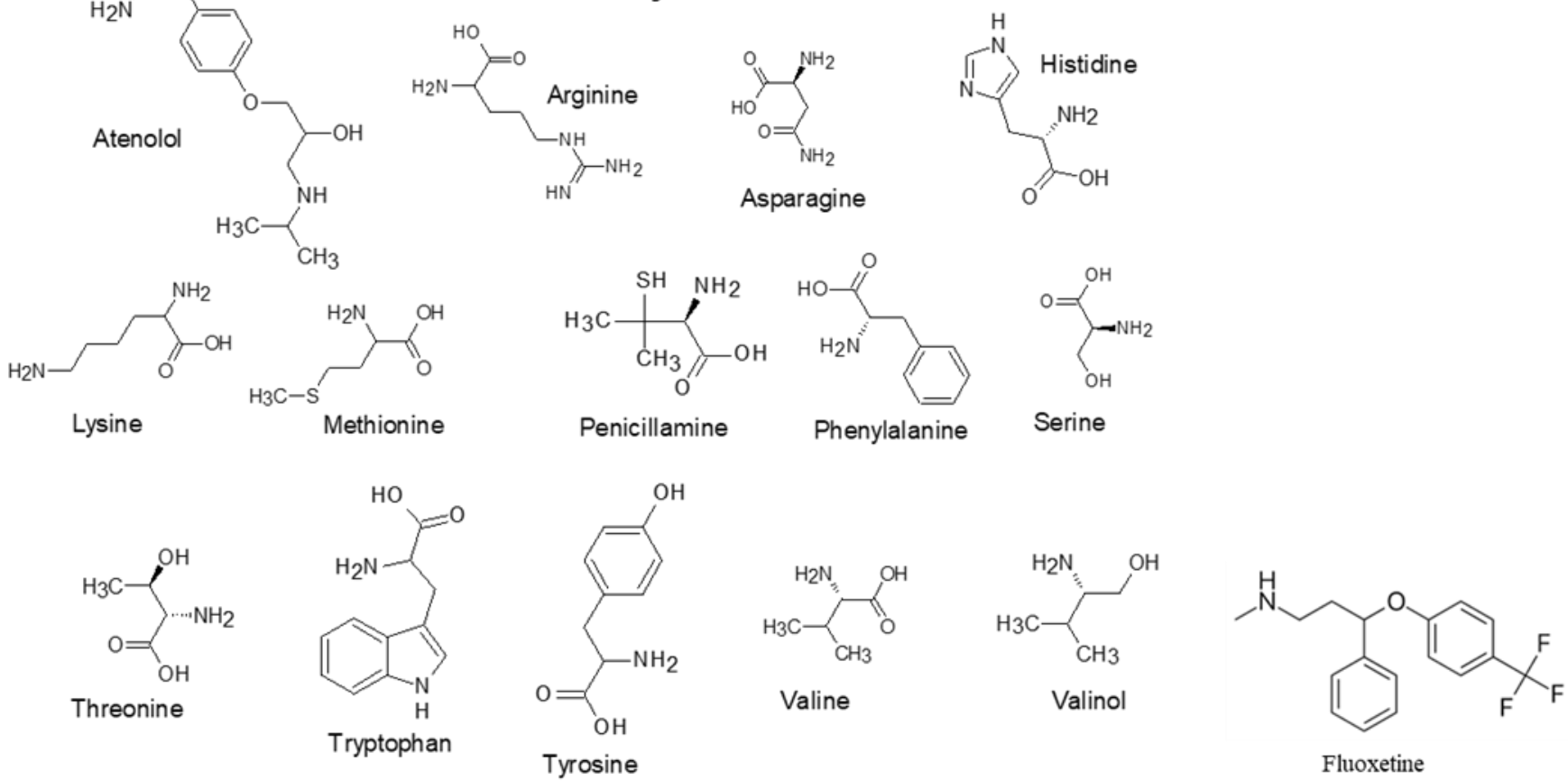

\section{Chiral selectors}

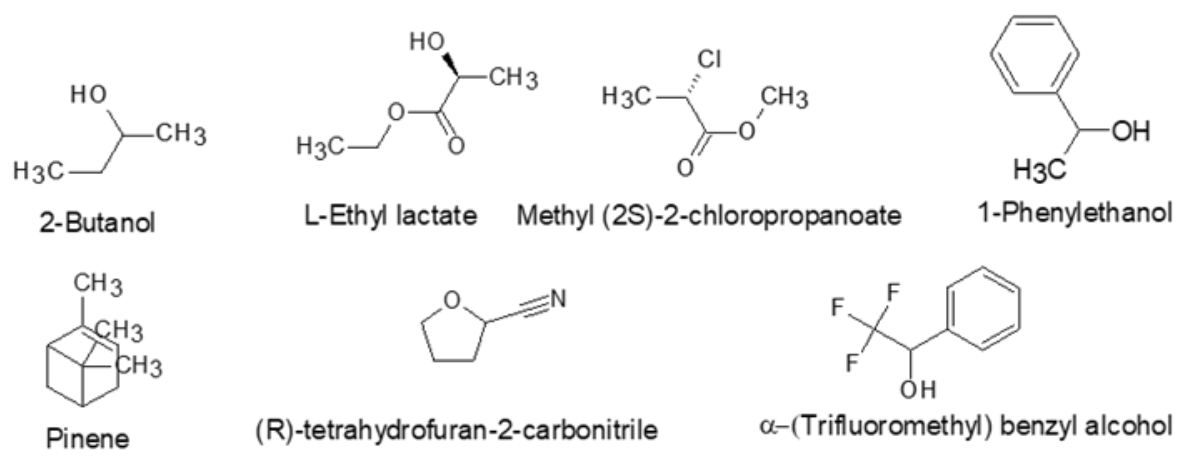

Figure S2. Chiral analytes and chiral selectors for chiral separation experiments 


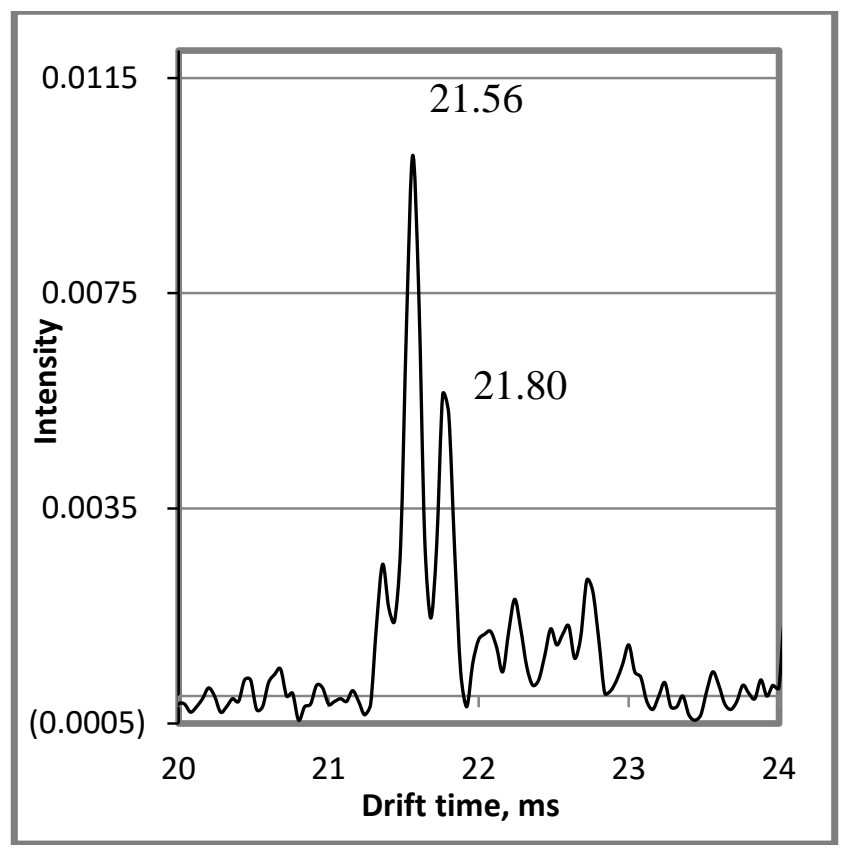

Figure S3. L-valinol IMS spectrum showing the resolution of the instrument and its capability to separate peaks separated by $0.24 \mathrm{~ms}$ or less. The peaks are separated by $0.24 \mathrm{~ms}$ and partially resolved. 


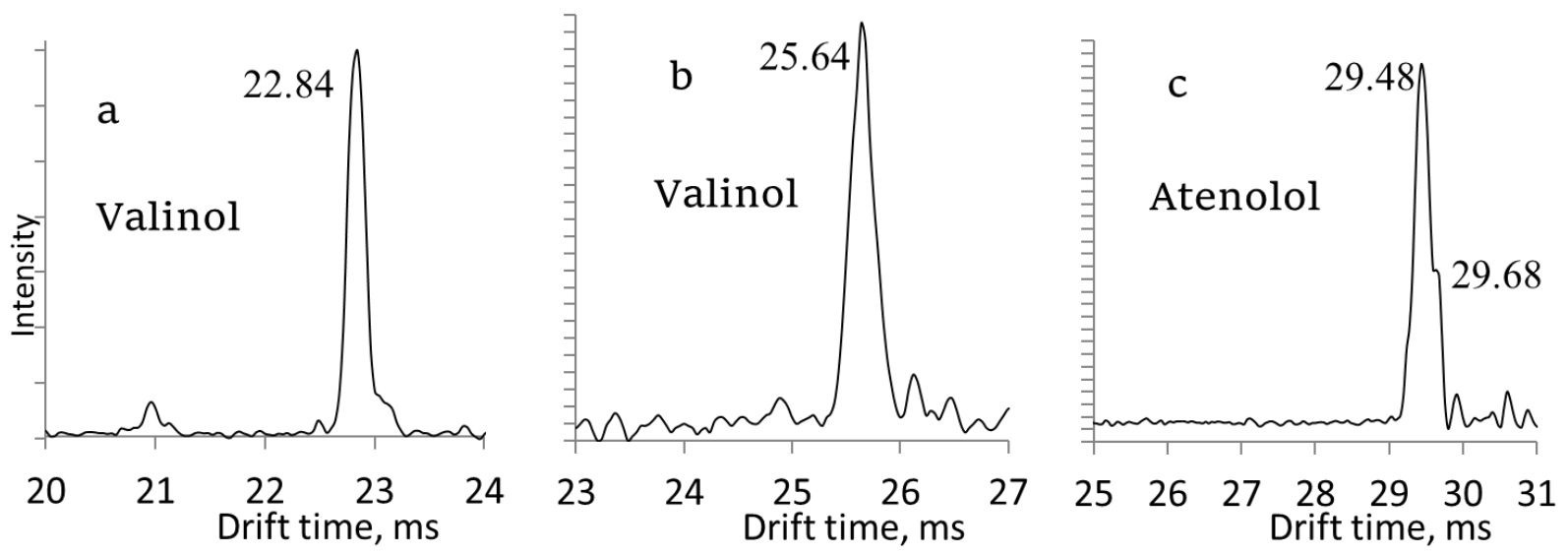

Figure S4. IMS spectra of racemic mixtures of 943- $\mu \mathrm{M}$ valinol at (S)-2-butanol flow rates of $45 \mu \mathrm{L} / \mathrm{hr}$ (a), $5 \mu \mathrm{L} / \mathrm{hr}$ (b), and (c) $100-\mu \mathrm{M}$ atenolol at a (S)-2-butanol flow rate of $50 \mu \mathrm{L} / \mathrm{hr}$ in the buffer gas. Temperature was $150^{\circ} \mathrm{C}$. No chiral separation is evident in these experiments. With the resolution shown in Figure $\mathrm{S} 3$ and the separation in drift times between the enantiomers in tables S5, 2, S6 and S7, there should be peaks partially resolved in these spectra. 

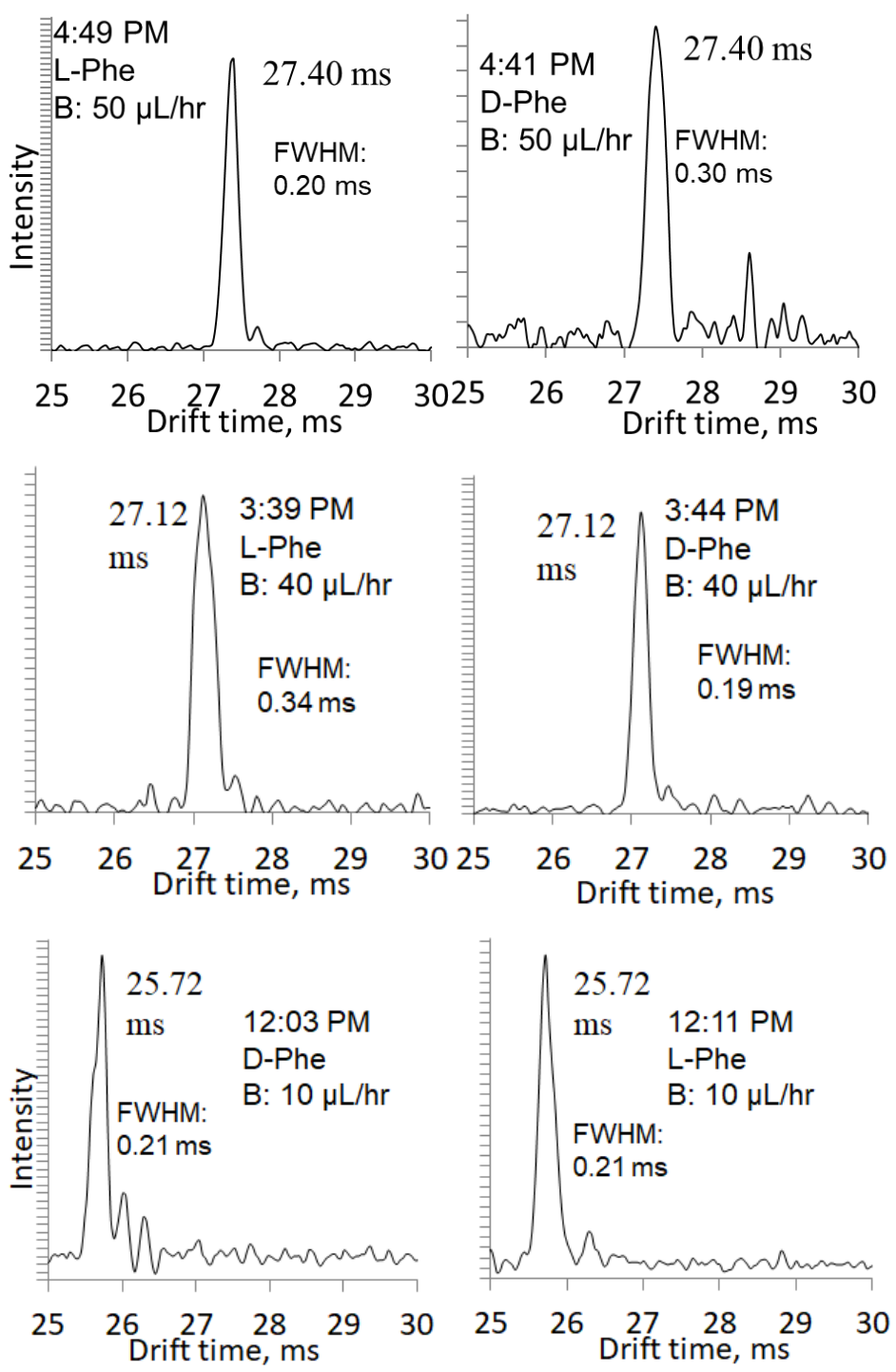

Figure S5. IMS spectra of the enantiomers D and L of phenylalanine when 50, 40, and 10 $\mu \mathrm{L} / \mathrm{hr}$ of 2 butanol (B) was introduced in the buffer gas at $150^{\circ} \mathrm{C}$. The time when the spectra was taken is shown. FWHM: full-width at half-maximum. With this data, resolving power was calculated in Table S10. 


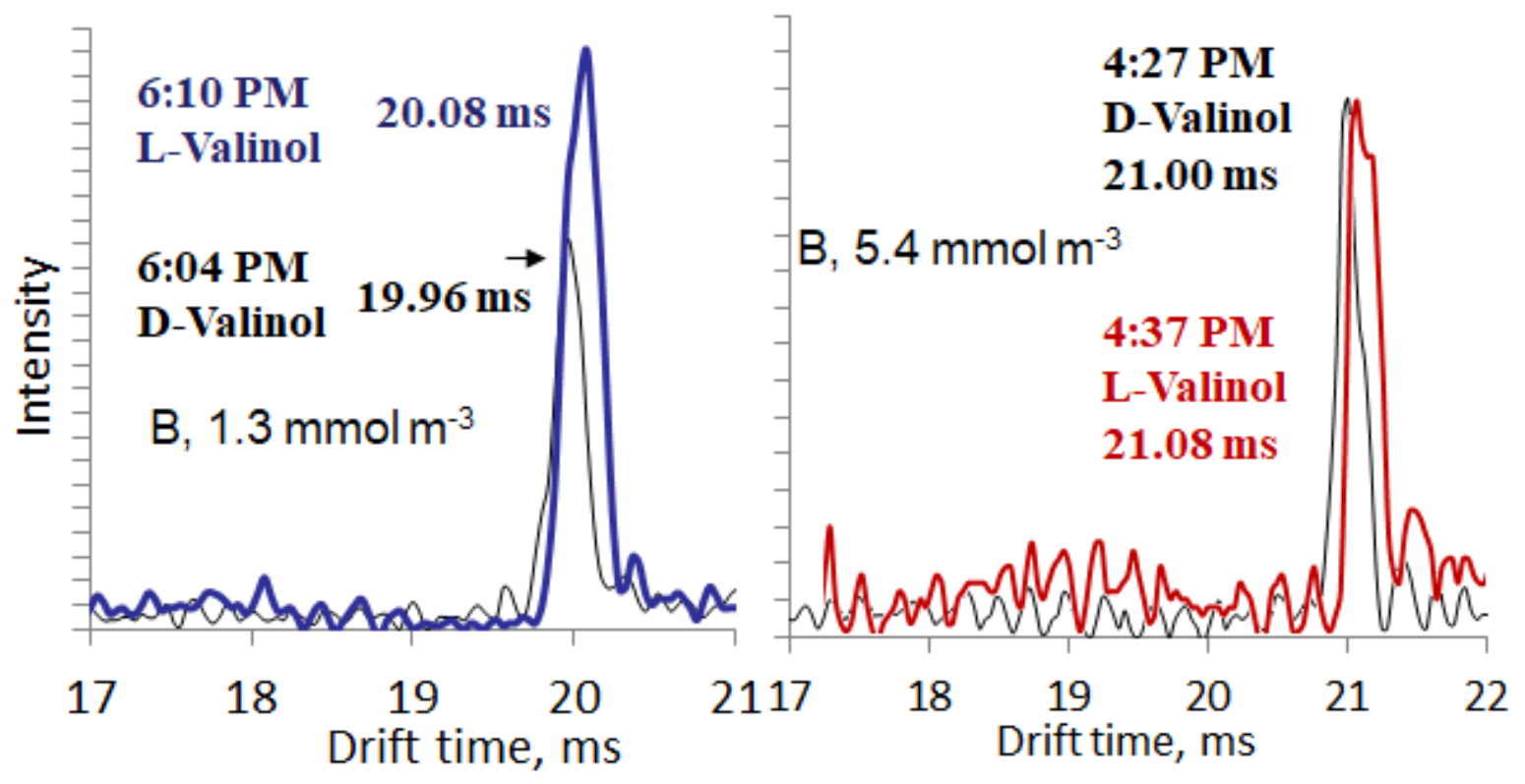

Figure S6a. Superimposed IMS spectra of valinol enantiomers at two concentrations of (S)-2butanol concentrations showing peaks with different drift times.

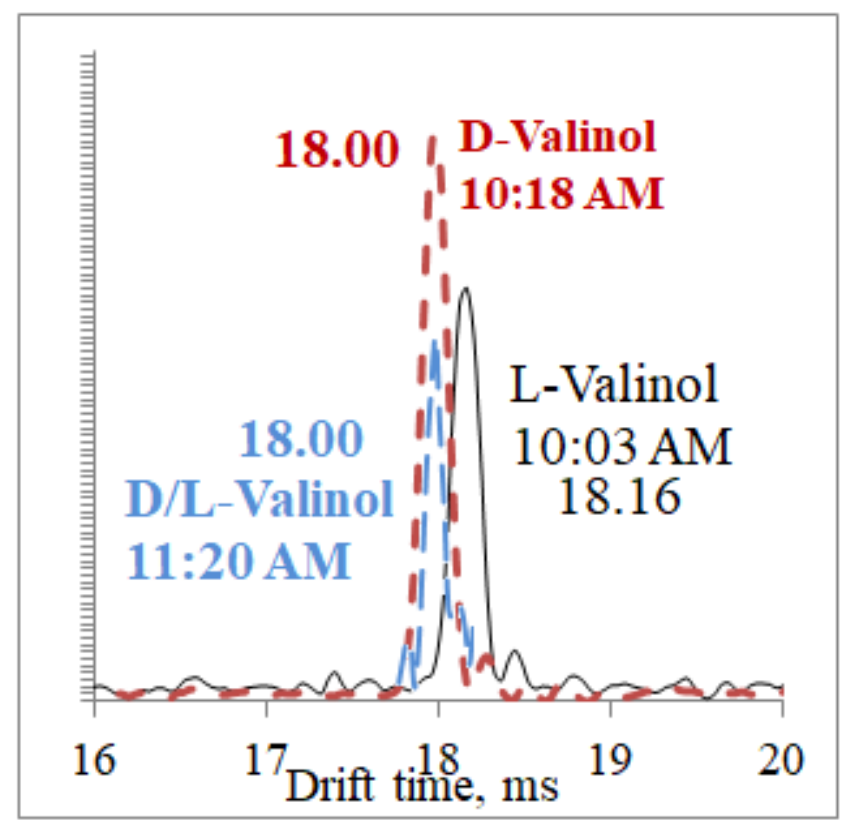

Figure S6b. Superimposed IMS spectra of valinol enantiomers (red dotted and black solid lines) and its racemic mixture (blue dotted line) at $1.3 \mathrm{mmol} \mathrm{m}^{-3}$ of (S)-2-butanol. The individual enantiomers had different drift times but the racemic mixture yielded a single peak. 


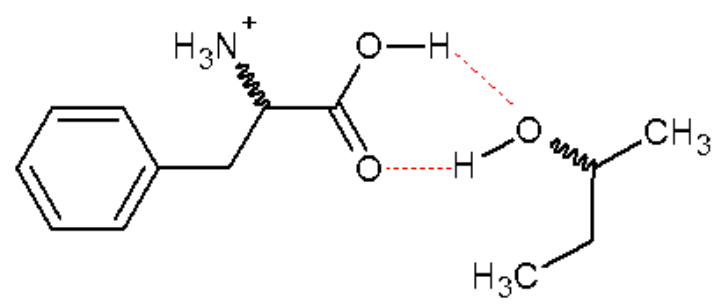

Interaction with the carboxylic group

Figure S7. Interaction phenylalanine:2-butanol

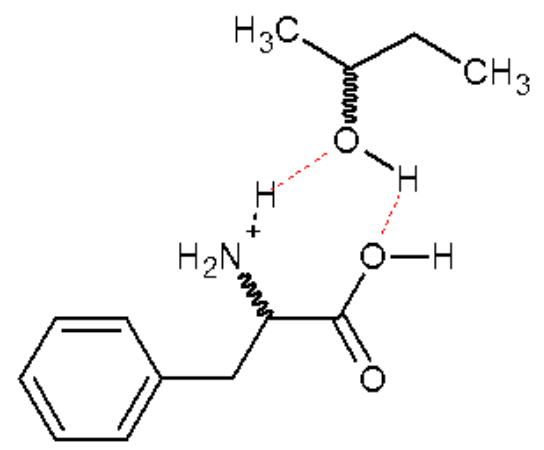

Interaction with the amine group 


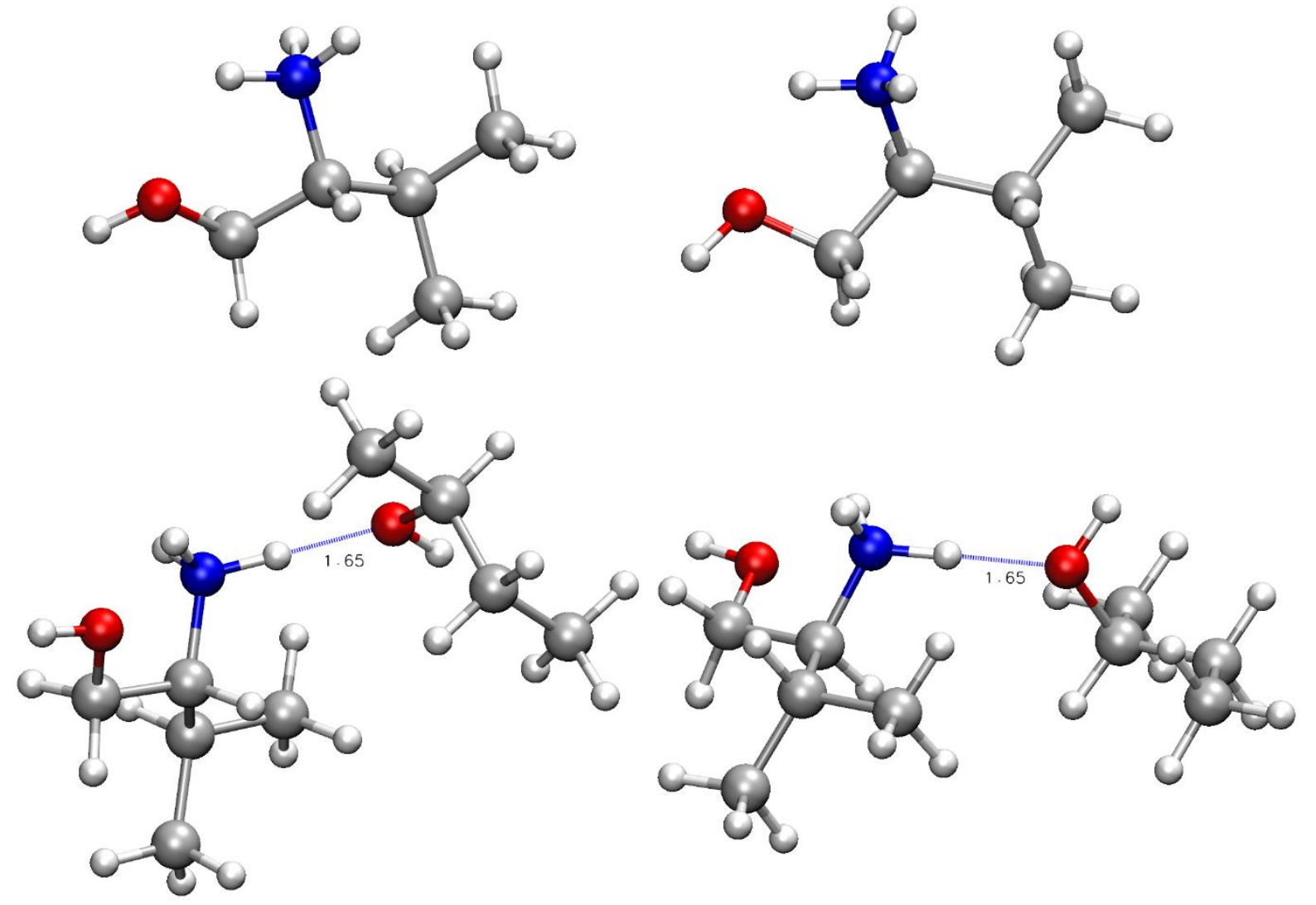

Figure S8. Isolated chiral valinol cations (above) and their complexes with 2-butanol (below). 


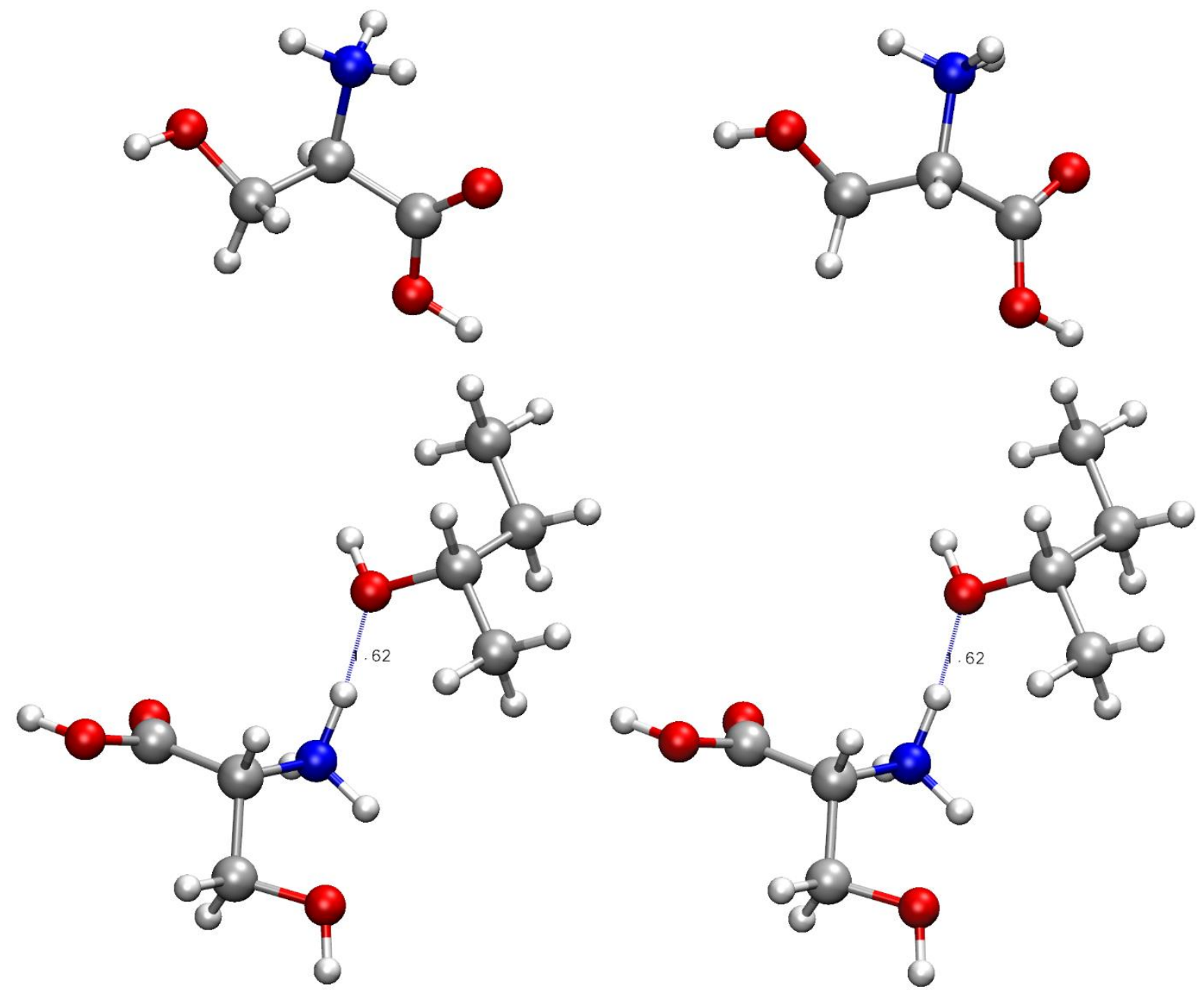

Figure S9. Isolated chiral serine cations (above) and their complexes with 2-butanol (below). 

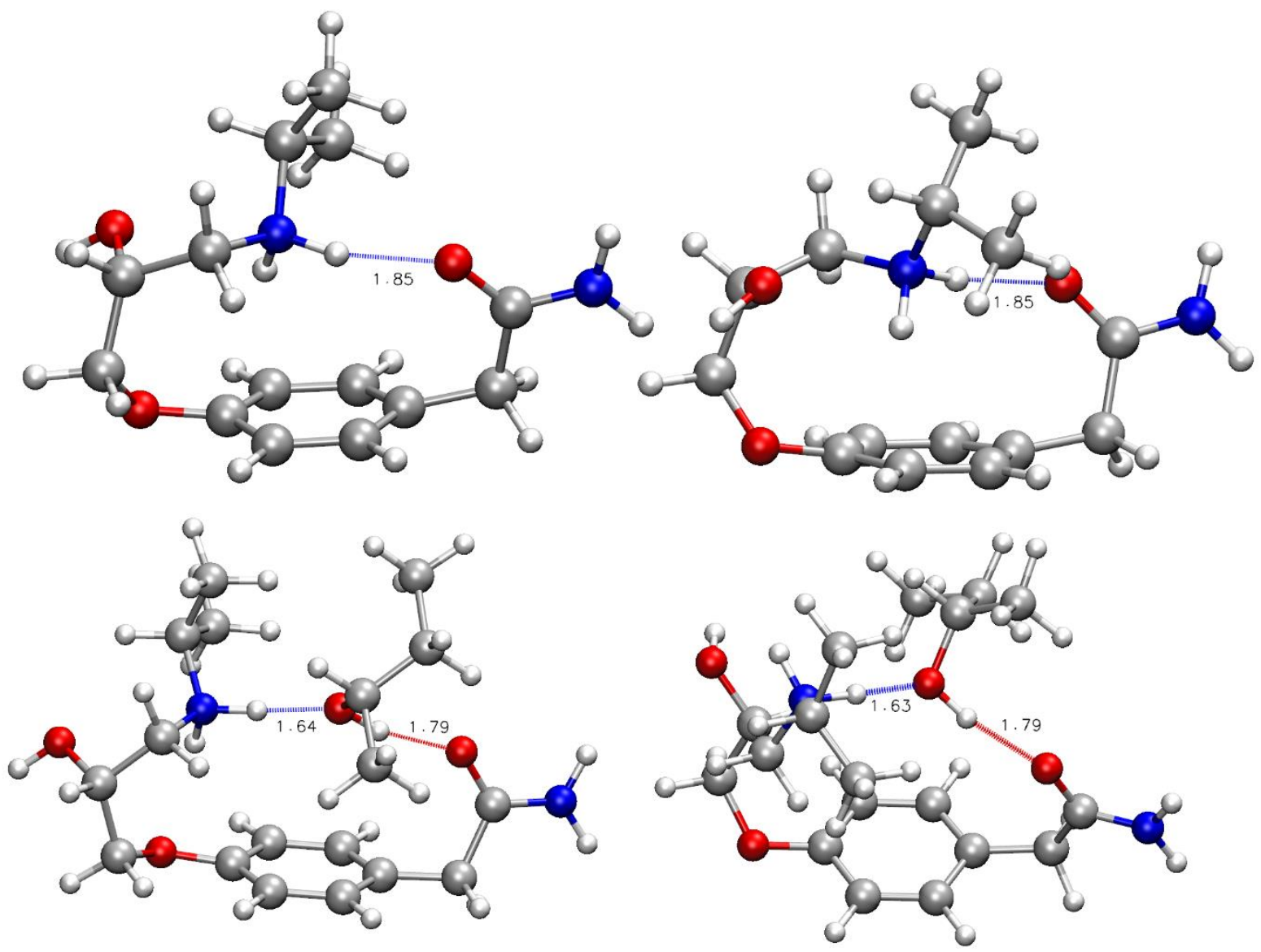

Figure S10. Isolated chiral atenolol cations (above) and their complexes with 2-butanol (below). 
Figure S11. Graphic explanation of Table 3. Mobility shifts of valinol enantiomers after the introduction of (S)-2-butanol in the buffer gas (drift time and flow rate data from reference $20)^{20}$.

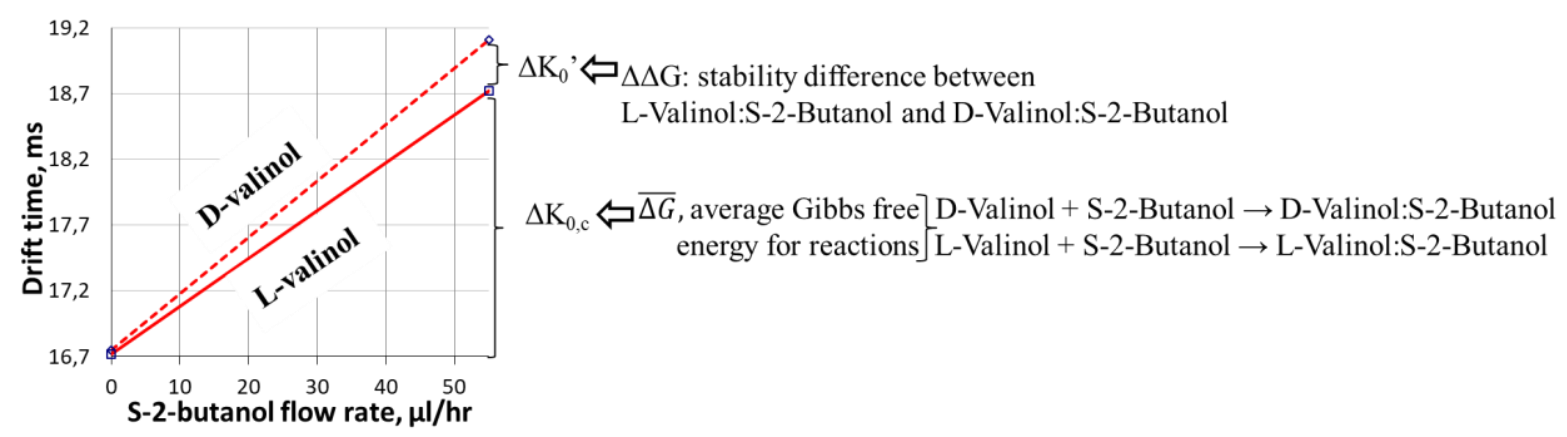

Mobility shifts such as $\Delta \mathrm{K}_{0, \mathrm{c}}$, the drift time shift when 2-butanol is injected in the buffer gas, and $\Delta \mathrm{K}_{0}$, the drift time separation between the $\mathrm{D}$ and $\mathrm{L}$ enantiomers, are produced by the analyte size increase after clustering with 2-butanol and the concomintant increase in collisions against the buffer gas. The energetics determine the lifespan of these clusters. Therefore, $\overline{\Delta G}$, the average Gibbs free energies for the formation reactions of L-Valinol:(S)-2-Butanol and DValinol:(S)-2-Butanol, determines $\Delta \mathrm{K}_{0, \mathrm{c} .} \Delta \Delta \mathrm{G}$, the difference of the stabilities between these two clusters, determines $\Delta \mathrm{K}_{0}$ '. The values of $\Delta \Delta \mathrm{G}$ and $\overline{\Delta G}$ must be proportional to the lifetime of the clusters and, consequently, to $\Delta \mathrm{K}_{0}$ ' and $\Delta \mathrm{K}_{0, \mathrm{c}}$, respectively; thus, $\frac{\Delta K_{0}{ }^{\prime}}{\overline{\Delta \Delta G}}$ and $\frac{\Delta K_{0, c}}{\overline{\Delta G}}$ must be similar. Because $\frac{\Delta K_{0}{ }^{\prime}}{\overline{\Delta \Delta G}}>\frac{\Delta K_{0, c}}{\overline{\Delta G}}, \Delta \mathrm{K}_{0}$ ' and $\Delta \Delta G$ must be relatively large and small, respectively, and the mobility shifts produced by the chiral interactions in the D-valinol:(S)-2-butanol complex would be relatively larger than those produced by non-chiral interactions. These results would be contrary to expectations because chiral interactions in these complexes are weaker than their strong non-chiral interactions, including the interaction with the formal charge on the cation. 
Table S1. Review of seven studies cited by Nagy et al. ${ }^{14}$ about enantiomer separations. Anyone of these studies performed enantiomer separation by only injecting a chiral selector. Most of the studies separated enantiomers through formation of chiral non-covalent diastereomeric complexes with other chiral molecules using transition metal cations.

\begin{tabular}{|l|l|c|}
\hline \multicolumn{1}{|c|}{ Analytes } & \multicolumn{1}{|c|}{ Method } & Reference \\
\hline $\begin{array}{l}\text { l-glucose, d- } \\
\text { glucose, l-allose, d- } \\
\text { allose, d-gulose, d- } \\
\text { galactose, and l- } \\
\text { mannose }\end{array}$ & $\begin{array}{l}\text { IMS-MS; monosaccharide enantiomers were } \\
\text { separated by IMS-MS as metal-bound trimeric } \\
\text { complexes with an amino acid or short amino acid } \\
\text { chain in its L form acting as chiral reference } \\
\text { compound }\end{array}$ & 21 \\
\hline Isobaric dipeptides & $\begin{array}{l}\text { IMS; crown ethers as shift reagents for non- } \\
\text { enatiomeric isobaric dipeptides }\end{array}$ & 22 \\
\hline Bile acids & $\begin{array}{l}\text { Separation of Bile acid isomers by formation of } \\
\text { cyclodextrin-bile acid host-guest inclusion } \\
\text { complex }\end{array}$ & 23 \\
\hline Amino acids & $\begin{array}{l}\text { Amino acid enantiomers were separated by FAIMS } \\
\text { as metal-bound trimeric complexes with another } \\
\text { amino acid in its L form acting as chiral reference } \\
\text { compound }\end{array}$ & 25 \\
\hline Amino acids & $\begin{array}{l}\text { TWIM-MS; cationisation with copper(II) and } \\
\text { multimer formation with D-proline }\end{array}$ & 26 \\
\hline Amino acids & $\begin{array}{l}\text { FAIMS; Diastereomeric proton bound complexes } \\
\text { were formed between enantiomers of amino acids } \\
\text { (analytes) and } N \text {-tert-butoxycarbonyl- } O \text {-benzyl-1- } \\
\text { serine }\end{array}$ & $\begin{array}{l}\text { IMS-MS; glycan isomers complexed with different } \\
\text { metal cations. }\end{array}$ \\
\hline $\begin{array}{l}\text { Non enantiomeric } \\
\text { glycans }\end{array}$ & $\begin{array}{l}\text { Determination of collisional cross sections for four } \\
\text { groups of isomeric carbohydrates as their group I } \\
\text { metal ion adducts } \\
\text { carbohydrates }\end{array}$ & 27 \\
\hline
\end{tabular}

TWIM: traveling wave ion mobility spectrometry.

FAIMS: High-field asymmetric waveform ion mobility.

21. Gaye, M. M.; Nagy, G.; Clemmer, D. E.; Pohl, N. L., Multidimensional Analysis of 16

Glucose Isomers by Ion Mobility Spectrometry. Anal Chem 2016, 88, 2335-44.

https://doi.org/10.1021/acs.analchem.5b04280

22. Hilderbrand, A. E.; Myung, S.; Clemmer, D. E., Exploring Crown Ethers as Shift

Reagents for Ion Mobility Spectrometry. Anal. Chem. 2006, 78, 6792-800.

$10.1021 / \mathrm{ac} 060439 \mathrm{v}$

23. Chouinard, C. D.; Cruzeiro, V. c. W. D.; Roitberg, A. E.; Yost, R. A., Rapid Ion Mobility

Separations of Bile Acid Isomers Using Cyclodextrin Adducts and Structures for Lossless

Ion Manipulations. J. Am. Soc. Mass Spectrom. 2017, 28, 323-331.

https://doi.org/10.1021/acs.analchem.8b02990

24. Mie, A.; Jornten-Karlsson, M.; Axelsson, B. O.; Ray, A.; Reimann, C. T., Enantiomer

Separation of Amino Acids by Complexation with Chiral Reference Compounds and High- 
Field Asymmetric Waveform Ion Mobility Spectrometry: Preliminary Results and Possible Limitations. Anal. Chem. 2007, 79, 2850-8. https://doi.org/10.1021/ac0618627

25. Domalain, V.; Hubert-Roux, M.; Tognetti, V.; Joubert, L.; Lange, C. M.; Rouden, J.; Afonso, C., Enantiomeric differentiation of aromatic amino acids using traveling wave ion mobility-mass spectrometry. Chem. Sci. 2014, 5, 3234-3239.

https://doi.org/10.1039/C4SC00443D

26. Zhang, J. D.; Mohibul Kabir, K. M.; Lee, H. E.; Donald, W. A., Chiral recognition of amino acid enantiomers using high-definition differential ion mobility mass spectrometry. Int. J. Mass Spectrom. 2018, 428, 1-7. https://doi.org/10.1016/j.ijms.2018.02.003 Zheng, X., Zhang, X., Schocker, N. S., Renslow, R. S., Orton, D. J., Khamsi, J., ... \& Baker, E. S. (2017). Enhancing glycan isomer separations with metal ions and positive and negative polarity ion mobility spectrometry-mass spectrometry analyses. Analytical and bioanalytical chemistry, 409(2), 467-476.

28. Huang, Y.; Dodds, E. D., Ion Mobility Studies of Carbohydrates as Group I Adducts: Isomer Specific Collisional Cross Section Dependence on Metal Ion Radius. Anal. Chem. 2013, 85, 9728-35. https://doi.org/10.1021/ac402133f 
Table S2. Racemic mixtures that could not be separated and individual enantiomers whose drift times were not significantly different, with several chiral selectors. There are 48 different unsuccessful experiments in this table. The analyte concentration was $500 \mu \mathrm{M}$ and the buffer gas temperature was $150^{\circ} \mathrm{C}$, unless otherwise specified. Data was obtained switching between one enantiomer to the other. See a detailed explanation of this table below.

\begin{tabular}{|c|c|}
\hline Chiral selector & $\begin{array}{l}\text { Analyte [Concentrations of chiral selector in the buffer gas in } \\
\qquad \mathrm{mmol} \mathrm{m}^{-3} \text { ] }\end{array}$ \\
\hline (R)-1-phenyl ethanol & D and L: Asn, Met, Phe: [5.3] and [2.1, 4.2, 6.3] \\
\hline & $\mathrm{D}$ and L-Phe $[0.42,0.63] ; \mathrm{R}$ and S-Atenolol[5.3] \\
\hline (R)-2-butanol & $\begin{array}{l}\text { D and L: Ser [6.9]; }{ }^{\mathrm{a}} \text { Met }[0.69],[4.8,6.2,7.6,14],[3.4,5.5,7.6] \\
\text { and }[1.4,2.8,4.1,5.5,6.9,8.3] ; \text { valinol, Val }[6.8](4 \text { experiments); } \\
\text { Tyr }[6.1] ; \mathrm{R} \text { and S-Ate }[13.5]\end{array}$ \\
\hline (S)-2-butanol & 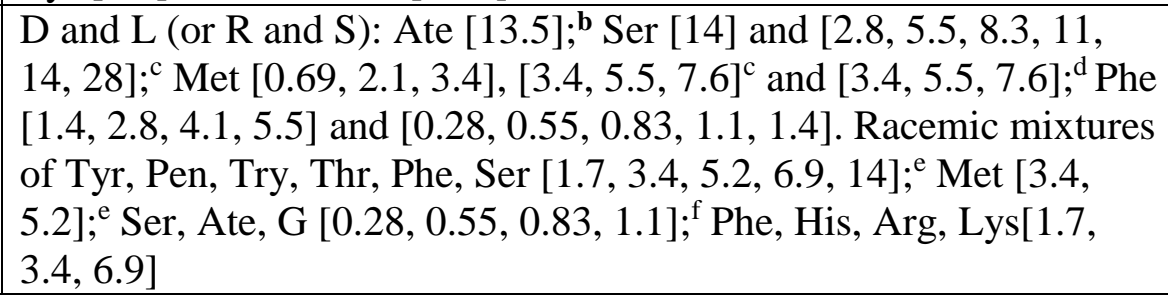 \\
\hline tFMBA & $\begin{array}{l}\text { mixtures of Ser, Thr, Met, Phe, Tyr, Try, Ate, valinol } \\
2,1.7,2.3]\end{array}$ \\
\hline (L)-ethyl lactate & $\mathrm{D}$ and $\mathrm{L}$ valinol $[3.3,6.6,13,26,55,110]$ \\
\hline $\begin{array}{l}\text { Methyl (S)-2- } \\
\text { chloropropionate }\end{array}$ & Valinol (D and L) and Atenolol (R and S) [0.11] \\
\hline \multicolumn{2}{|c|}{$\begin{array}{l}\text { Buffer gas temperatures of } 110,140,170 \text { and } 200^{\circ} \mathrm{C} \text { were tested; }{ }^{\mathrm{b}} 4 \text { experiments on two days } \\
\text { Two experiments on two days; }{ }^{\mathrm{d}} \text { Buffer gas temperature was } 138^{\circ} \mathrm{C} ;{ }^{\mathrm{e}} \text { Buffer gas temperature } \\
\text { f } 100,150,200 \text {, and } 250^{\circ} \mathrm{C} \text { were tested, two experiments on different days; }{ }^{\mathrm{f}} \text { analyte } \\
\text { concentrations of } 50,250 \text { and } 1000 \mu \mathrm{M} \text { were tested. Several of these experiments included } \\
\text { epetitions at different hours. Arg: arginine, Ate: atenolol, G: glucose, His: histidine, Lys } \\
\text { ysine, Met: methionine, Pen: penicillamine, Phe: phenylalanine, Ser: serine, tFMBA: (R)- } \alpha \text { - } \\
\text { trifluoromethyl) benzyl alcohol, Thr: threonine, Try: tryptophan, Tyr: tyrosine, Val: valine }\end{array}$} \\
\hline
\end{tabular}

Table S2 summarizes the following experiments:

- (R)-1-phenyl ethanol was used as the chiral selector at concentrations of $5.3 \mathrm{mmol} \mathrm{m}^{-3}$ in the buffer gas for the analysis of $500 \mu \mathrm{M}$ individual solutions of the enantiomers of asparagine, methionine, and phenylalanine at $150^{\circ} \mathrm{C}$; additionally, in other experiment, these amino acids were tested at 2.1, 4.2, and $6.3 \mathrm{mmol} \mathrm{m}^{-3}$ of (R)-1-phenyl ethanol in the same experimental conditions.

- (S)-1-phenyl ethanol was used as the chiral selector at 0.42 and $0.63 \mathrm{mmol} \mathrm{m}^{-3}$ in the buffer gas for the analysis of 500- $\mu \mathrm{M}$ individual solutions of the enantiomers of phenylalanine and atenolol at $150^{\circ} \mathrm{C}$; in other experiment, individual enantiomers of atenolol were tested at $5.3 \mathrm{mmol} \mathrm{m}^{-3}$ of (S)-1-phenyl ethanol in the same experimental conditions.

- (R)-2-butanol was used as the chiral selector at $0.69 \mathrm{mmol} \mathrm{m}^{-3} ; 4.8,6.2,7.6$ and $14 \mathrm{mmol}$ $\mathrm{m}^{-3} ; 3.4,5.5,7.6 \mathrm{mmol} \mathrm{m}^{-3}$; and 1.4, 2.8, 4.1, 5.5, 6.9, $8.3 \mathrm{mmol} \mathrm{m}^{-3}$ in the buffer gas for 
the analysis of $500-\mu \mathrm{M}$ individual solutions of the enantiomers of methionine at $150^{\circ} \mathrm{C}$ in four different experiments; in other experiment, in the same experimental conditions, the following 500- $\mu \mathrm{M}$ racemic mixtures were analyzed: atenolol with $13.5 \mathrm{mmol} \mathrm{m}^{-3}$ of (R)2-butanol in the buffer gas, serine with $6.9 \mathrm{mmol} \mathrm{m}^{-3}$ of (R)-2-butanol in the buffer gas at 4 different temperatures: $110,140,170$ and $200^{\circ} \mathrm{C}$, tyrosine with $6.1 \mathrm{mmol} \mathrm{m}^{-3}(\mathrm{R})-2$ butanol in the buffer gas, and valinol and valine, in 4 experiments on different days, with $6.8 \mathrm{mmol} \mathrm{m}^{-3}$ of (R)-2-butanol in the buffer gas.

- When (S)-2-butanol was used as the chiral selector, the following individual enantiomers were analyzed: $500-\mu \mathrm{M}$ solutions of $\mathrm{R}$ and S-atenolol with $13.5 \mathrm{mmol} \mathrm{m}^{-3}$ of (S)-2-butanol in the buffer gas in 4 experiments during two days; $250-\mu \mathrm{M}$ solutions of $\mathrm{D}$ and L-serine with $2.8,5.5,8.3,11$, and $14 \mathrm{mmol} \mathrm{m}^{-3}$ of (S)-2-butanol in two different experiments; 500$\mu \mathrm{M}$ methionine at $0.69,2.1$, and $3.4 \mathrm{mmol} \mathrm{m}^{-3}$ of (S)-2-butanol in one experiment, 3.4, 5.5, and $7.6 \mathrm{mmol} \mathrm{m}^{-3}$ of (S)-2-butanol in two additional experiments on two different days at two temperatures, 150 and $138^{\circ} \mathrm{C}$, respectively; $500-\mu \mathrm{M}$ D and L-phenylalanine at 1.4, 2.8, 4.1 , and $5.5 \mathrm{mmol} \mathrm{m}^{-3}$ of (S)-2-butanol, and $0.28,0.55,0.83,1.1,1.4 \mathrm{mmol} \mathrm{m}^{-3}$ of (S)-2butanol in other experiment; Also, the following racemic mixtures were analyzed: tyrosine, penicillamine, tryptophan, threonine, phenylalanine, and serine with 1.7, 3.4, 5.2, 6.9, 14 mmol m${ }^{-3}$ of (S)-2-butanol in the buffer gas at $100,150,200$, and $250^{\circ} \mathrm{C}$, in two experiments on different days; methionine at 3.4 and $5.2 \mathrm{mmol} \mathrm{m}^{-3}$ of (S)-2-butanol in the buffer gas at $100,150,200$, and $250^{\circ} \mathrm{C}$, in two experiments on different days; 50, 250 and $1000 \mu \mathrm{M}$ solutions of serine, atenolol, and glucose at $0.28,0.55,0.83$, and $1.1 \mathrm{mmol} \mathrm{m}^{-3}$ of (S)-2butanol with several repetitions at different hours; and, finally, phenylalanine, histidine, arginine, and lysine at 1.7, 3.4, $6.9 \mathrm{mmol} \mathrm{m}^{-3}$ of (S)-2-butanol.

- (R)- $\alpha$-(trifluoromethyl) benzyl alcohol was used as the chiral selector at 0.3, 0.55, 1.1, 1.7, and $2.3 \mathrm{mmol} \mathrm{m}^{-3}$ in the buffer gas for the analyses of $500-\mu \mathrm{M}$ racemic mixtures of serine, threonine, methionine, phenylalanine, tyrosine, tryptophan, atenolol, and valinol, at $150^{\circ} \mathrm{C}$.

- Ethyl lactate was used as the chiral selector at 3.3, 6.6, 13, 26, 55, and $110 \mathrm{mmol} \mathrm{m}^{-3}$ in the buffer gas for the analisis of $500-\mu \mathrm{M}$ solutions of the enantiomers of valinol, at $150^{\circ} \mathrm{C}$.

- Methyl (S)-2-chloropropionate was used as the chiral selector at $0.09 \mathrm{mmol} \mathrm{m}^{-3}$ in the buffer gas for the análisis of 500- $\mu \mathrm{M}$ solutions of the enantiomers of valinol and atenolol, at $150^{\circ} \mathrm{C}$. 
Table S3. Drift times of valine enantiomers in an 8-hr period using (S)-2-butanol chiral selector. Solutions of D- valine $(\boldsymbol{\square})$, L- valine $(\diamond)$ and racemic mixtures of valine $(\Delta)$ at a 943 $\mu \mathrm{M}$ concentration were analyzed when $0.69 \mathrm{mmol} \mathrm{m}^{-3}$ of $(\mathrm{S})$-2-butanol was introduced into the buffer gas at $150{ }^{\circ} \mathrm{C}$. The drift times were different for both enantiomers (average drift time separation of $\sim 0.3 \mathrm{~ms}$ ) although the racemic mixtures yielded only one peak, with a drift time similar to that of D-valine. Variations in drift time for every enantiomer were caused mainly by changes in atmospheric pressure. The drift times of the enantiomers were statistically different $(\mathrm{P}<0.05)$. The data for this table is plotted in Figure 1.

\begin{tabular}{|c|c|c|c|c|c|}
\hline Cation & RSD & $\mathbf{d t}$ & SD & $\mathbf{n}$ & $\begin{array}{c}\text { Experiment } \\
\text { sequence }\end{array}$ \\
\hline L & 0.103 & 19.42 & 0.02 & 4 & $1^{\circ}$ \\
\hline D & 0.156 & 19.23 & 0.03 & 10 & $2^{\circ}$ \\
\hline L & 0.102 & 19.53 & 0.02 & 7 & $3^{\circ}$ \\
\hline D & 0.104 & 19.20 & 0.02 & 5 & $4^{\circ}$ \\
\hline D/L & 0.208 & 19.26 & 0.04 & 3 & $5^{\circ}$ \\
\hline L & 0.205 & 19.50 & 0.04 & 6 & $6^{\circ}$ \\
\hline D & 0.207 & 19.30 & 0.04 & 6 & $7^{\circ}$ \\
\hline L & 0.153 & 19.58 & 0.03 & 4 & $8^{\circ}$ \\
\hline D & 0.149 & 19.42 & 0.03 & 5 & $9^{\circ}$ \\
\hline D/L & 0.157 & 19.16 & 0.03 & 4 & $10^{\circ}$ \\
\hline L & 0.206 & 19.41 & 0.04 & 5 & $11^{\circ}$ \\
\hline D & 0.208 & 19.22 & 0.04 & 5 & $12^{\circ}$ \\
\hline L & 0.103 & 19.38 & 0.02 & 5 & $13^{\circ}$ \\
\hline
\end{tabular}

dt: drift time, ms; SD: standard deviation; n: number of experiments 
Table S4. Effect of chiral selector concentration on the mobilities of valinol enantiomers at 125 and $200^{\circ} \mathrm{C} .943-\mu \mathrm{M}$ solutions of D-valinol ( $\square$ ), L-valinol $(\diamond)$ and racemic mixtures

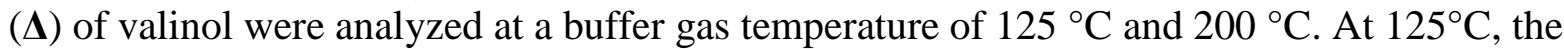
enantiomers had the same drift time but the racemic mixture always yielded a single peak. At $200^{\circ} \mathrm{C}$, the enantiomers had the same drift time because the interaction valinol-(S)-2-butanol decreased by the weak analyte-ligand bonds at high temperature and the difference in drift time between the enantiomers was too small. The drift times were statistically different $(\mathrm{P}<0.05)$ when $(\mathrm{S})$-2-butanol was introduced at $125^{\circ} \mathrm{C}$ (bold text). The data for this table is plotted in Figure 5.

\begin{tabular}{|c|c|c|c|c|c|c|}
\hline $125^{\circ} \mathrm{C}$ & & & & & & \\
\hline $\begin{array}{c}\text { (S)-2-butanol } \\
\mathrm{mmol} / \mathrm{m}^{-3}\end{array}$ & dt-D-Valinol & dt-L-Valinol & $\begin{array}{c}\text { Average } \\
\text { SD }\end{array}$ & $\mathbf{n}$ & $\begin{array}{l}\text { dt-D/L- } \\
\text { Valinol } \\
\end{array}$ & $\mathbf{n}$ \\
\hline 0 & 20.21 & 20.21 & 0.04 & 5 & & \\
\hline 0.4 & 21.49 & 21.89 & 0.03 & 8 & 21.54 & 4 \\
\hline 1.7 & 23.60 & 23.91 & 0.02 & 6 & 24.26 & 5 \\
\hline 3.4 & 24.25 & 24.48 & 0.04 & 6 & & \\
\hline 5.1 & 24.63 & 24.79 & 0.05 & 8 & & \\
\hline 6.8 & 24.80 & 25.00 & 0.04 & 5 & & \\
\hline \multicolumn{7}{|l|}{$200^{\circ} \mathrm{C}$} \\
\hline $\begin{array}{c}\text { (S)-2-butanol } \\
\mathrm{mmol} / \mathrm{m}^{-3}\end{array}$ & dt-D-Valinol & dt-L-Valinol & $\begin{array}{c}\text { Average } \\
\text { SD }\end{array}$ & $\mathbf{n}$ & $\begin{array}{l}\text { dt-D/L- } \\
\text { Valinol } \\
\end{array}$ & $\mathbf{n}$ \\
\hline 0 & 17.20 & 17.21 & 0.03 & 4 & & \\
\hline 0.4 & 17.24 & 17.24 & 0.03 & 5 & 17.25 & 5 \\
\hline 1.7 & 17.27 & 17.27 & 0.04 & 7 & 17.26 & 4 \\
\hline 3.4 & 17.29 & 17.29 & 0.02 & 3 & & \\
\hline 5.1 & 17.30 & 17.29 & 0.03 & 4 & 17.30 & 3 \\
\hline 6.8 & 17.30 & 17.30 & 0.02 & 6 & & \\
\hline
\end{tabular}

dt: drift time, ms; SD: standard deviations for D and L enantiomers; n: number of experiments. Mobilities were measured for both enantiomers alternating between the $\mathrm{R}$ and $\mathrm{S}$ species. 
Table S5. Chiral experiments with $D$ and $L$ phenylalanine; (R)-1-phenyl ethanol was used as chiral selector between 0 and $11 \mathrm{mmol} \mathrm{m}^{-3}$ at $\mathbf{1 7 5}^{\circ} \mathrm{C}$. A difference in drift times was obtained when the pure enantiomers were individually electrosprayed into the buffer gas alternating between the $\mathrm{R}$ and $\mathrm{S}$ species. Standard deviations and averages were calculated for three or more measurements.

\begin{tabular}{|c|c|c|c|c|c|c|}
\hline $\begin{array}{l}\text { (R)-1-phenyl } \\
\text { ethanol, mmol m-3 }\end{array}$ & L-Phe & $\mathbf{n}$ & D-Phe & $\mathbf{n}$ & $\begin{array}{l}\text { Drift time } \\
\text { difference } \\
\quad(m s)\end{array}$ & $\begin{array}{c}\text { Significance } \\
\text { level }\end{array}$ \\
\hline 0 & $23.63 \pm 0.02$ & 3 & $23.64 \pm 0.02$ & 4 & 0.01 & - \\
\hline 5.3 & $24.83 \pm 0.02$ & 8 & $24.75 \pm 0.03$ & 4 & 0.08 & $P=0.0002$ \\
\hline 7.9 & $25.12 \pm 0.00$ & 4 & $25.05 \pm 0.01$ & 4 & 0.07 & $\mathrm{P}=0.0001$ \\
\hline 11 & $25.35 \pm 0.04$ & 4 & $25.28 \pm 0.02$ & 8 & 0.07 & $P=0.002$ \\
\hline
\end{tabular}

The values were obtained as follows: $23.63,23.64,24.83,24.75,25.05,25.12,25.04$ (repetition of the 25.05 measurement); 25.28, 25.35, 25.26 (repetition of the 25.28 measurement). 
Table S6. Chiral experiments with D and L-valinol; (S)-2-butanol was used as chiral selector between 0 and $5.5 \mathrm{mmol} \mathrm{m}^{-3}$ at $150^{\circ} \mathrm{C}$. The pure enantiomers were individually electrosprayed into the buffer gas alternating between the $\mathrm{R}$ and $\mathrm{S}$ species. With the resolution shown in Figure $\mathrm{S} 3$ and the separation in drift times between the enantiomers in this table, there should be peaks partially resolved in these experiments when the racemic mixture was injected but only one peak was obtained. The drift times in bold text were statistically different $(\mathrm{P}<0.05)$.

\begin{tabular}{|c|c|c|c|}
\hline $\begin{array}{c}\text { (S)-2-Butanol } \\
\text { concentration, } \\
\text { mmol m }^{-3}\end{array}$ & \multicolumn{2}{|c|}{ Drift time, ms } & \multirow{2}{*}{$\Delta \mathrm{dt}$} \\
\cline { 2 - 3 } & $\mathrm{L}$ & $\mathrm{D}$ & \\
\hline 0 & 18.94 & 18.94 & 0.00 \\
\hline 0.14 & $\mathbf{1 9 . 1 9}$ & $\mathbf{1 9 . 4 0}$ & 0.21 \\
\hline 0.69 & $\mathbf{1 9 . 5 7}$ & $\mathbf{1 9 . 9 4}$ & 0.37 \\
\hline 1.4 & 19.96 & 20.02 & 0.06 \\
\hline 2.8 & $\mathbf{2 0 . 2 9}$ & $\mathbf{2 0 . 4 2}$ & 0.13 \\
\hline 4.1 & 20.83 & 20.80 & -0.03 \\
\hline 5.5 & $\mathbf{2 1 . 1 1}$ & $\mathbf{2 1 . 0 4}$ & -0.07 \\
\hline
\end{tabular}


Table S7. Effect of temperature on the mobilities of valinol enantiomers. $943-\mu \mathrm{M}$ solutions of D-valinol and L-valinol were analyzed when $0.28 \mathrm{mmol} \mathrm{m}^{-3}$ of (S)-2-butanol was introduced into the buffer gas between 80 and $200^{\circ} \mathrm{C}$. The pure enantiomers were individually electrosprayed into the buffer gas alternating between the $\mathrm{R}$ and $\mathrm{S}$ species. With the resolution shown in Figure S3 and the separation in drift times between the enantiomers in this table, there should be peaks partially resolved in these experiments when the racemic mixture was injected but only one peak was obtained. The drift times for the $\mathrm{D}$ and L-enantiomers were significantly different according to the $\mathrm{P}$ values. Other experimental conditions were as in Figure 3.

\begin{tabular}{|c|c|c|c|c|c|c|c|}
\hline & \multicolumn{3}{|c|}{ L } & \multicolumn{3}{c|}{ D } & \multirow{2}{*}{ P } \\
\cline { 1 - 7 } T, ${ }^{\circ} \mathbf{C}$ & dt & SD & n & dt & SD & n & \\
\hline 80 & 29.73 & 0.05 & 4 & 29.75 & 0.04 & 5 & 0.52 \\
\hline 100 & 26.20 & 0.05 & 6 & 26.69 & 0.08 & 7 & $<0.0001$ \\
\hline 110 & 23.48 & 0.05 & 5 & 23.5 & 0.03 & 5 & 0.47 \\
\hline 170 & 21.22 & 0.04 & 4 & 21.52 & 0.09 & 5 & 0.0005 \\
\hline 200 & 19.68 & 0.05 & 5 & 19.84 & 0.01 & 4 & 0.0004 \\
\hline
\end{tabular}


Table S8. Effect of temperature on the mobilities of valinol enantiomers (data for Figure 5). $943-\mu \mathrm{M}$ solutions of D-valinol, L-valinol, and racemic mixtures of valinol were analyzed when $0.28 \mathrm{mmol} \mathrm{m}^{-3}$ of (S)-2-butanol was introduced into the buffer gas from 125 to $200^{\circ} \mathrm{C}$. The racemic mixtures yielded only one peak, with a drift time close to that of D-valinol. Other experimental conditions were as in Figure 1. The drift times of the enantiomers were statistically different $(\mathrm{P}<0.05)$.

\begin{tabular}{|c|c|c|c|c|c|c|c|c|c|c|c|c|}
\hline \multirow{2}{*}{$\mathrm{T},{ }^{\circ} \mathbf{C}$} & \multicolumn{4}{|c|}{ D-valinol } & \multicolumn{4}{|c|}{ L-valinol } & \multicolumn{4}{|c|}{ D/L-valinol } \\
\hline & $\mathrm{dt}, \mathrm{ms}$ & SD dt & $\mathrm{K}_{0}$ & $S D K_{0}$ & $\mathrm{dt}, \mathrm{ms}$ & SD dt & Ko & SD $K_{0}$ & $\mathrm{dt}, \mathrm{ms}$ & SD dt & $\mathrm{K}_{0}$ & $S D K_{0}$ \\
\hline 125 & 23.35 & 0.07 & 1.631 & 0.02 & 23.46 & 0.06 & 1.616 & 0.02 & - & - & - & \\
\hline 150 & 19.23 & 0.03 & 1.814 & 0.02 & 19.42 & 0.02 & 1.796 & 0.02 & 19.26 & 0.04 & 1.811 & 0.02 \\
\hline 175 & 17.98 & 0.02 & 1.838 & 0.02 & 18.15 & 0.02 & 1.821 & 0.02 & 17.99 & 0.02 & 1.838 & 0.02 \\
\hline 200 & 16.50 & 0.00 & 1.890 & 0.03 & 16.58 & 0.02 & 1.881 & 0.03 & 16.50 & 0.01 & 1.890 & 0.03 \\
\hline
\end{tabular}

dt: drift time, ms; SD: standard deviation; $n$ : number of experiments 
Table S9. $\Delta K_{0}$ ' and $\Delta K_{0, c}$ : values for experiments from Table 1 in reference $20 .{ }^{20} \Delta K_{0}$ ' was calculated as the percentage difference between the mobilities of both enantiomers in (S)-2butanol-doped buffer gas. $\Delta \mathrm{K}_{0, c}$ : percentage change in $K_{0}$ when butanol flow rate was varied from 0 to $65 \mu \mathrm{L} / \mathrm{hr}$ into the buffer gas. $\mathrm{r}=0.24$ and 0.45 for the mass- $\Delta \mathrm{K}_{0}$ ' and mass- $\Delta \mathrm{K}_{0, \mathrm{c}}$ correlations, respectively.

\begin{tabular}{|c|c|c|c|c|c|c|c|}
\hline \multirow[b]{2}{*}{ Mass } & \multirow[b]{2}{*}{ Species } & \multirow{2}{*}{$\begin{array}{c}\text { Ko value } \\
\text { in } N_{2}\end{array}$} & \multicolumn{2}{|c|}{$\mathrm{K}_{0}$ in $(\mathrm{S})$-2-butanol } & \multirow{2}{*}{$\begin{array}{c}\Delta \mathbf{K}_{\mathbf{0}}{ }^{\prime} \\
\%\end{array}$} & \multicolumn{2}{|c|}{$\Delta \mathrm{K}_{0, \mathrm{c}} \%$} \\
\hline & & & D-ee & L-ee & & D-ee $\#$ & L-ee ${ }^{*}$ \\
\hline 263.3 & Atenolol & 1.18 & $1.18^{\#}$ & $1.16^{*}$ & 1.7 & 0.0 & -1.7 \\
\hline 204.2 & Tryptophan & 1.32 & 1.25 & 1.23 & 1.6 & -5.3 & -6.8 \\
\hline 149.2 & Methionine & 1.56 & 1.23 & 1.22 & 0.8 & -21.2 & -21.8 \\
\hline 119.1 & Threonine & 1.69 & 1.51 & 1.48 & 2.0 & -10.7 & $\begin{array}{ll}-12.4 \\
\end{array}$ \\
\hline 217.2 & $\begin{array}{c}\text { Methyl } \alpha- \\
\text { glucopyranoside }{ }^{\&}\end{array}$ & 1.30 & 1.15 & 1.12 & 2.6 & -11.5 & -13.8 \\
\hline 203.2 & Glucose $^{\&}$ & 1.30 & 1.23 & 1.21 & 1.6 & -5.4 & -6.9 \\
\hline 149.2 & Penicillamine & 1.53 & 1.42 & 1.40 & 1.4 & -7.2 & -8.5 \\
\hline 103.2 & Valinol & 1.74 & 1.62 & 1.60 & 1.2 & -6.9 & -8.0 \\
\hline 165.2 & Phenylalanine & 1.45 & 1.31 & 1.28 & 2.3 & -9.7 & -11.7 \\
\hline 105.1 & Serine & 1.73 & 1.55 & 1.52 & 1.9 & -10.4 & -12.1 \\
\hline
\end{tabular}

ee: enantiomer. ${ }^{\#}$ R-enantiomer for atenolol; ${ }^{*}$ S-enantiomer for atenolol. For example: the value 1.7 for the $\Delta \mathrm{K}_{0}$ 'of atenolol was calculated as [(1.18-1.16)/1.18]*100 and the value -8.0 for the $\Delta \mathrm{K}_{0, \mathrm{c}}$ for L- valinol was calculated as [(1.60-1.74)/1.74]*100. Mass: mass of the protonated species. Mass: mass of the sodiated ${ }^{\&}$ (glucose and methyl $\alpha$-glucopyranoside) or protonated species. 
Table S10. Resolving power of the IMS instrument for selected peaks of phenylalanine. The average resolving power was 116. This data was calculated with IMS spectra in Figure S5. The time when the spectra was taken is shown.

\begin{tabular}{|c|c|c|c|c|c|}
\hline Cation & $\mathbf{d t}(\mathbf{m s})$ & $\mathbf{B}, \boldsymbol{\mu l} / \mathbf{h r}$ & $\mathbf{F W H M}$ & $\mathbf{R p}$ & Time \\
\hline L-Phe & 27.40 & 50 & 0.20 & 137 & $16: 49$ \\
\hline D-Phe & 27.40 & 50 & 0.30 & 91 & $16: 41$ \\
\hline L-Phe & 27.12 & 40 & 0.34 & 80 & $15: 39$ \\
\hline D-Phe & 27.12 & 40 & 0.19 & 143 & $15: 44$ \\
\hline L-Phe & 25.72 & 10 & 0.21 & 122 & $12: 11$ \\
\hline D-Phe & 25.72 & 10 & 0.21 & 122 & $12: 03$ \\
\hline
\end{tabular}

dt: drift time; B: (S)-2-butanol flow rate; Rp: resolving power; FWHM: full-width at halfmaximum. $10 \mu \mathrm{L} / \mathrm{hr}=1.35 \mathrm{mmol} \mathrm{m}^{-3}$ 